Report no. 99/04

\title{
A posteriori error analysis for stabilised finite element approximations of transport problems
}

\author{
Paul Houston \\ Oxford University Computing Laboratory, Wolfson Building, \\ Parks Road, Oxford OX1 3QD, UK \\ Rolf Rannacher \\ Institut für Angewandte Mathematik, Universität Heidelberg, \\ Im Neuenheimer Feld 293, D-69120 Heidelberg, Germany. \\ Endre Süli \\ Oxford University Computing Laboratory, Wolfson Building, \\ Parks Road, Oxford OX1 3QD, UK
}

\begin{abstract}
We develop the a posteriori error analysis of stabilised finite element approximations to linear transport problems via duality arguments. Two alternative dual problems are considered: one is based on the formal adjoint of the hyperbolic differential operator, the other on the transposition of the bilinear form for the stabilised finite element method. We show both analytically and through numerical experiments that the second approach is superior in the sense that it leads to sharper a posteriori error bounds and more economical adaptively refined meshes.
\end{abstract}

Subject classifications: AMS(MOS): 65N12, 65N15, 65N30

Key words and phrases: Stabilised finite element methods, a posteriori error analysis, hyperbolic problems

P. Houston and E. Süli acknowledge the financial support of the EPSRC (Grant GR/K76221).

R. Rannacher acknowledges the financial support of the German Research Association (DFG) through the SBF 359 "Reactive Flow, Diffusion and Transport", University of Heidelberg.

Oxford University Computing Laboratory

Numerical Analysis Group

Wolfson Building

Parks Road

Oxford, England OX1 3QD

March, 1999 


\section{Introduction}

The purpose of this article is to develop the a posteriori error analysis of stabilised finite element approximations to transport problems, including the streamline diffusion finite element method and the least-squares stabilised finite element method (see [9]). For simplicity, we restrict the discussion to the case of a scalar linear transport equation of the form

$$
\beta \cdot \nabla u+c u=f
$$

subject to appropriate inflow boundary condition. Generalisations to systems and nonlinear equations are possible and will be remarked on where appropriate.

Mesh adaptation in finite element discretisations should be based on rigorous a posteriori error estimates; for hyperbolic equations such estimates should reflect the inherent mechanisms of error propagation (see $[6,8]$ ). These considerations are particularly important when local quantities such as point values, local averages or flux integrals of the analytical solution are to be computed with high accuracy. Selective error estimates of this kind can be obtained by the optimal control technique proposed in [5] and [2] which is based on duality arguments analogous to those from the a priori error analysis of finite element methods. In the resulting a posteriori error estimates the element-residuals of the computed solution are multiplied by local weights involving the dual solution. These weights represent the sensitivity of the relevant error quantity with respect to variations of the local mesh size. Since the dual solution is usually unknown analytically, it has to be approximated numerically. On the basis of the resulting a posteriori error estimate the current mesh is locally adapted and then new approximations to the primal and the dual solution are computed. This feed-back process is repeated, for instance, until the required error tolerance is reached. In this way, optimal meshes can be obtained for various kinds of error measures, where optimal can mean most economical for achieving a prescribed accuracy TOL or most accurate for a given maximum number $N_{\max }$ of mesh cells. This approach is quite universal as it can, in principle, be applied to almost any problem, linear or nonlinear, as long as it is posed in a variational setting. For a collection of examples, we refer to the survey article [13].

A new feature of this technique, when applied to stabilised finite element methods, is the choice of the dual problem, and it is the study of this question that represents the subject of the present paper. In particular, we show that the naive approach of using the natural dual based on the formal adjoint of the partial differential operator results in $a$ posteriori error estimates in which the stabilisation terms may strongly dominate the other residual terms, leading to over-refinement of the mesh. We then show that this deficiency can be overcome by exploiting the dual of the 'stabilised' primal differential operator. In this way, extra powers of the mesh size are gained in the stabilisation terms, resulting in an optimal-order error bound. The success of the latter approach is explained by theoretical analysis and is illustrated in detail by numerical experiments.

The outline of the paper is as follows. We start, in Section 2, by describing the key ideas on a one-dimensional model problem. In Section 3, guided by the one-dimensional analysis, we then consider the question of a posteriori error estimation for stabilised Galerkin approximations to multi-dimensional transport equations. In particular, we discuss the 
problem of a posteriori error estimation for norms as well as linear functionals of the solution. Section 4 presents a series of numerical experiments which illustrate and verify the theoretical results. Finally, in Section 5 we summarise the work presented in this paper and draw some conclusions.

\section{The analysis of the one-dimensional problem}

In order to highlight the key issues concerning the error analysis while avoiding unnecessary technicalities, we begin by considering the following model problem:

$$
u^{\prime}(x)=f(x), \quad \text { for } x \in(0,1], \quad u(0)=g,
$$

where $f \in L_{2}(0,1)$ and $g$ is a given real number; it is a simple matter to show that this has a unique solution in $H^{1}(0,1)$.

Now suppose that the interval $[0,1]$ has been partitioned by a nonuniform mesh defined by the mesh-points $\left\{x_{i}\right\}_{i=0}^{N}$, where $0=x_{0}<x_{1}<\ldots<x_{N-1}<x_{N}=1$, and $N$ is a positive integer, $N \geq 1$. We let $I_{j}=\left(x_{j-1}, x_{j}\right], h_{j}=x_{j}-x_{j-1}$, and introduce the mesh function $h$ defined on $[0,1]$ by $h(x)=h_{j}$ for $x \in I_{j}, j=1, \ldots, N$. On this partition we consider the finite element space $S_{h}, S_{h} \subset H^{1}(0,1)$, consisting of continuous piecewise polynomials of fixed degree $p, p \geq 1$. We recall the following standard approximation property of the finite element interpolant $P_{h}: H^{1}(0,1) \rightarrow S_{h}$ (see [4]):

(A) Given that $v \in H^{s+1}(0,1)$ for some $s, 0 \leq s \leq p$, there exists a constant $C_{i}$, independent of $v$ and $h$, such that

$$
\left\|v-P_{h} v\right\|_{L_{2}\left(I_{j}\right)}+h_{j}\left|v-P_{h} v\right|_{H^{1}\left(I_{j}\right)} \leq C_{i} h_{j}^{s+1}|v|_{H^{s+1}\left(I_{j}\right)}
$$

for all $j=1, \ldots, N$. Here, $|\cdot|_{H^{r}\left(I_{j}\right)}$ denotes the usual seminorm of the Sobolev space $H^{r}\left(I_{j}\right), r \geq 1$.

Next, we introduce the streamline diffusion finite element approximation of (2.1); to do so, we consider a positive function $\delta \in L_{\infty}(0,1)$ called the streamline diffusion parameter, the bilinear form $B_{\delta}(\cdot, \cdot)$ defined by

$$
B_{\delta}(w, v)=\left(w^{\prime}, v+\delta v^{\prime}\right)+w(0) v(0),
$$

and the linear functional $l_{\delta}$ given by

$$
l_{\delta}(v)=\left(f, v+\delta v^{\prime}\right)+g v(0) .
$$

In these definitions $(\cdot, \cdot)$ denotes the inner product of $L_{2}(0,1)$.

The streamline diffusion method is: find $u_{h} \in S_{h}$ such that

$$
B_{\delta}\left(u_{h}, v_{h}\right)=l_{\delta}\left(v_{h}\right) \quad \forall v_{h} \in S_{h} .
$$

As $B_{\delta}\left(v_{h}, v_{h}\right)>0$ for all $v_{h}$ in $S_{h} \backslash\{0\}$ and $S_{h}$ is a finite-dimensional linear space, it follows that (2.3) has a unique solution $u_{h}$ in $S_{h}$. Formally, (2.3) can be viewed as a perturbation of the standard Galerkin method corresponding to $\delta \equiv 0$. 
Here we shall be concerned with the a posteriori error analysis of the streamline diffusion method: our aim is to derive a bound on the global error $e=u-u_{h}$ in terms of the computable finite element residuals

$$
r_{h}=f-u_{h}^{\prime}, \quad r_{h}^{-}=g-u_{h}(0),
$$

which arise from inserting $u_{h}$ into the differential equation in (2.1) and the associated boundary condition at inflow. The analysis relies on the following Galerkin property:

$$
B_{\delta}\left(u-u_{h}, v_{h}\right)=0 \quad \forall v_{h} \in S_{h}
$$

The identity (2.4) is easily seen to hold by noting (2.3) and that

$$
B_{\delta}\left(u, v_{h}\right)=l_{\delta}\left(v_{h}\right) \quad \forall v_{h} \in S_{h} .
$$

In the next two subsections we shall describe two distinct approaches to the a posteriori error analysis of the streamline diffusion method; being driven by duality arguments, they both proceed in the same manner, but since they use different dual problems the resulting a posteriori error bounds are not identical.

\subsection{The first approach and its limitations}

The starting point in the argument is the following dual problem: given that $\psi \in C^{\infty}[0,1]$, find $z$ such that

$$
-z^{\prime}=\psi(x), \quad x \in[0,1), \quad z(1)=0
$$

clearly, this has a unique solution $z \in C^{\infty}[0,1]$. Now we are ready to state our first result.

Lemma 1 The following bound holds for $0 \leq s \leq p$ :

$$
\begin{array}{r}
\left|\left(u-u_{h}, \psi\right)\right| \leq C_{i} \sum_{j=1}^{N}\left\|h r_{h}\right\|_{L_{2}\left(I_{j}\right)} \min _{0 \leq \sigma \leq s}\left\{h_{j}^{\sigma}|z|_{H^{\sigma+1}\left(I_{j}\right)}\right\} \\
+\left(1+C_{i}\right) \sum_{j=1}^{N}\left\|\delta r_{h}\right\|_{L_{2}\left(I_{j}\right)}|z|_{H^{1}\left(I_{j}\right)}
\end{array}
$$

where $C_{i}$ is the constant from (2.2).

Proof: Recalling the dual problem (2.5), integrating by parts, and appealing to the Galerkin property (2.4), we deduce that, for any $z_{h} \in S_{h}$,

$$
\left(u-u_{h}, \psi\right)=\left(r_{h}, z-z_{h}\right)-\left(\delta r_{h}, z_{h}^{\prime}\right)+r_{h}^{-}\left(z(0)-z_{h}(0)\right) \equiv \mathrm{I}+\mathrm{II}+\mathrm{III} .
$$

Next, given that $s$ is a fixed real number, $0 \leq s \leq p$, we make a specific choice of $z_{h}$ : we take $z_{h}=P_{h} z$, where $P_{h}$ is the finite element interpolant of $v$ from $S_{h}$. Then, $z(0)-z_{h}(0)=$ 
$z(0)-\left(P_{h} z\right)(0)=0$, yielding III $=0$. It remains to estimate terms I and II. Let us first deal with term I. Noting (2.2), we have, for any $\sigma_{j} \in[0, s], j=1, \ldots, N$,

$$
|\mathrm{I}| \leq \sum_{j=1}^{N}\left\|h_{j}^{\sigma_{j}+1} r_{h}\right\|_{L_{2}\left(I_{j}\right)}\left\|h_{j}^{-\left(\sigma_{j}+1\right)}\left(z-P_{h} z\right)\right\|_{L_{2}\left(I_{j}\right)} \leq C_{i} \sum_{j=1}^{N}\left\|h r_{h}\right\|_{L_{2}\left(I_{j}\right)} h_{j}^{\sigma_{j}}|z|_{H^{\sigma_{j}+1}\left(I_{j}\right)}
$$

Consequently,

$$
|I| \leq C_{i} \sum_{j=1}^{N}\left\|h r_{h}\right\|_{L_{2}\left(I_{j}\right)} \min _{0 \leq \sigma \leq s}\left\{h_{j}^{\sigma}|z|_{H^{\sigma+1}\left(I_{j}\right)}\right\} .
$$

For term II, on writing $P_{h} z=z+\left(P_{h} z-z\right)$ and applying (2.2) we have

$$
|\mathrm{II}| \leq \sum_{j=1}^{N}\left\|\delta r_{h}\right\|_{L_{2}\left(I_{j}\right)}\left\|\left(P_{h} z\right)^{\prime}\right\|_{L_{2}\left(I_{j}\right)} \leq\left(1+C_{i}\right) \sum_{j=1}^{N}\left\|\delta r_{h}\right\|_{L_{2}\left(I_{j}\right)}|z|_{H^{1}\left(I_{j}\right)} .
$$

Collecting the bounds on I and II and inserting them into (2.7), we complete the proof of the estimate (2.6).

In order to ensure that the first term in this a posteriori error bound fully reflects the approximation properties of the test space, one would wish to choose $s$ as large as possible, namely $s=p$. However, since in the second term $r_{h}$ is multiplied by $\delta$ rather than $h^{s+1}$, the error bound (2.6) will be properly balanced only if $\delta \leq C_{\delta} h^{s+1}$ where $C_{\delta}$ is a fixed positive constant. For $s>0$, such choice of $\delta$ is considerably smaller than the standard one of $\delta=C_{\delta} h$ (cf. [9], [10]), and may lead to an under-stabilised numerical approximation which exhibits non-physical numerical oscillations. Thus, from the practical point of view, there appears to be no benefit from choosing any value of $s$ other than the suboptimal one of $s=0$; indeed, with $s=0$ in (2.6) and noting that $|z|_{H^{1}(0,1)}=\|\psi\|_{L_{2}(0,1)}$, we immediately deduce the next result.

Corollary 2 The following a posteriori error bound holds:

$$
\left\|u-u_{h}\right\|_{L_{2}(0,1)} \leq C_{i}\left\|h r_{h}\right\|_{L_{2}(0,1)}+\left(1+C_{i}\right)\left\|\delta r_{h}\right\|_{L_{2}(0,1)} .
$$

In the next section, we present a strategy which overcomes the undesirable features of this first approach.

\subsection{The second approach}

Although (2.5) is the most natural candidate for the dual problem, it is by no means the only possible choice. Indeed, as will be seen below, the a posteriori error bound (2.6) can be improved by selecting a dual problem which respects the particular structure of the Galerkin property (2.4).

Thus, we now consider the following dual problem: given that $\psi$ is in $C^{\infty}[0,1]$, find $z_{\delta}$ in $H^{1}(0,1)$ such that

$$
B_{\delta}\left(w, z_{\delta}\right)=(w, \psi) \quad \forall w \in H^{1}(0,1)
$$


Here, the solution to the dual problem is denoted by $z_{\delta}$ in order to emphasise the dependence on the stabilisation parameter $\delta$. Recalling the definition of $B_{\delta}$, a simple calculation based on integration by parts shows that $(2.8)$ can be restated as follows:

$$
\begin{aligned}
-\left(z_{\delta}+\delta z_{\delta}^{\prime}\right)^{\prime} & =\psi(x), & & x \in \bigcup_{j=1}^{N}\left(x_{j-1}, x_{j}\right) \\
{\left[z_{\delta}+\delta z_{\delta}^{\prime}\right]\left(x_{j}\right) } & =0, & & j=1, \ldots, N-1 \\
z_{\delta}^{\prime}(0) & =0, & & \left(z_{\delta}+\delta z_{\delta}^{\prime}\right)(1)=0
\end{aligned}
$$

where $[w](x)=w(x+)-w(x-)$ denotes the jump of $w$ at $x$. In particular, when $\delta \in$ $W_{\infty}^{1}(0,1)$, the function $z_{\delta}+\delta z_{\delta}^{\prime}$ is continuous on $[0,1]$ and the jump conditions are trivially satisfied. In this case, the dual problem collapses to

$$
\begin{aligned}
-\left(z_{\delta}+\delta z_{\delta}^{\prime}\right)^{\prime} & =\psi(x), \quad x \in(0,1), \\
z_{\delta}^{\prime}(0) & =0, \quad\left(z_{\delta}+\delta z_{\delta}^{\prime}\right)(1)=0 .
\end{aligned}
$$

To motivate the hypotheses which we shall make on $\psi$, we give the following characterisation of the dual solution.

Lemma 3 Suppose that $\delta$ is a constant, $\delta>0$, and let $n$ be a nonnegative integer; then

$$
\begin{aligned}
z_{\delta}(x)= & \int_{x}^{1} \psi(\xi) \mathrm{d} \xi+\sum_{\mathrm{m}=1}^{\mathrm{n}}(-1)^{\mathrm{m}-1} \delta^{\mathrm{m}}\left[\psi^{(\mathrm{m}-1)}(\mathrm{x})-e^{-\mathrm{x} / \delta} \psi^{(\mathrm{m}-1)}(0)\right] \\
& +(-1)^{n} \delta^{n} e^{-x / \delta} \int_{0}^{x} \psi^{(n)}(\xi) e^{\xi / \delta} \mathrm{d} \xi
\end{aligned}
$$

When $n=0$ the index set of the summation in the second term on the right is empty, and the corresponding sum is then defined to be identically zero.

PROOF: Let $n=0$; integrating the differential equation in (2.9) between $x$ and 1 we deduce, recalling the boundary condition at $x=1$, that

$$
z_{\delta}+\delta z_{\delta}^{\prime}=\int_{x}^{1} \psi(\xi) \mathrm{d} \xi, \quad \mathrm{x} \in[0,1]
$$

Solving the differential equation (2.11) gives

$$
z_{\delta}(x) \mathrm{e}^{x / \delta}-z_{\delta}(0)=\int_{0}^{x} \chi(\xi)\left(\mathrm{e}^{\xi / \delta}\right)^{\prime} \mathrm{d} \xi, \quad \text { where } \quad \chi(\mathrm{x})=\int_{\mathrm{x}}^{1} \psi(\xi) \mathrm{d} \xi
$$

Integrating by parts on the right and noting the boundary condition at $x=0$ in (2.9), which, according to $(2.11)$, is equivalent to demanding that $z_{\delta}(0)=\chi(0)$, we deduce that

$$
z_{\delta}(x)=\chi(x)+\mathrm{e}^{-x / \delta} \int_{0}^{x} \psi(\xi) \mathrm{e}^{\xi / \delta} \mathrm{d} \xi
$$

which is just the required identity for $n=0$. 
For $n \geq 1$, we write under the integral sign on the right in the last equality

$$
\mathrm{e}^{\xi / \delta}=\delta^{n} \frac{\mathrm{d}^{\mathrm{n}}}{\mathrm{d} \xi^{\mathrm{n}}} \mathrm{e}^{\xi / \delta}
$$

and integrate by parts $n$ times to deduce (2.10).

It is clear from this lemma that derivatives of $z_{\delta}$ will exhibit a boundary layer at $x=0$, unless the function $\psi$ and its derivatives vanish at this point. Armed by this observation, we state the following result.

Lemma 4 a) Let $\delta \in W_{\infty}^{1}(0,1)$ and suppose that there exists a constant $K_{1}$ such that

$$
1+\frac{1}{2} \delta^{\prime}(x) \geq K_{1}>0 \quad \text { for a.e. } x \in(0,1) ;
$$

then, there is a positive constant $M_{1}=M_{1}\left(K_{1}\right)$ such that, for each $\psi \in C^{\infty}[0,1]$,

$$
\left|z_{\delta}\right|_{H^{1}(0,1)} \leq M_{1}\|\psi\|_{L_{2}(0,1)} .
$$

b) Let $0 \leq m \leq p-1, \delta \in W_{\infty}^{m+2}(0,1)$ and suppose that there exist constants $L_{m+2}$ and $K_{m+2}$ such that $\left|\delta^{(m+2)}(x)\right| \leq L_{m+2}$ for a.e. $x \in(0,1)$ and

$$
1+\left(m+\frac{3}{2}\right) \delta^{\prime}(x) \geq K_{m+2}>0 \quad \text { for a.e. } x \in(0,1)
$$

then, there exists a positive constant $M_{m+2}=M_{m+2}\left(K_{m+2}, M_{1}, \ldots, M_{m+1}, L_{2}, \ldots, L_{m+2}\right)$ such that for each $\psi \in C^{\infty}[0,1]$ with $\psi^{(l)}(0)=0$ for all $l=0, \ldots, m$, we have

$$
\left|z_{\delta}\right|_{H^{m+2}(0,1)} \leq M_{m+2}|\psi|_{H^{m+1}(0,1)} .
$$

Proof: a) To prove (2.12), we differentiate (2.11) to deduce that

$$
\left(1+\delta^{\prime}\right) z_{\delta}^{\prime}+\delta z_{\delta}^{\prime \prime}=-\psi(x), \quad x \in(0,1] .
$$

Taking the $L_{2}(0,1)$ inner product of $(2.14)$ with $z_{\delta}^{\prime}$ and noting that $z_{\delta}^{\prime}(0)=0$, upon manipulating the second term on the left and applying the Cauchy-Schwarz inequality on the right, we arrive at

$$
K_{1}\left\|z_{\delta}^{\prime}\right\|_{L_{2}(0,1)}^{2}+\frac{1}{2} \delta(1)\left|z_{\delta}^{\prime}(1)\right|^{2} \leq\|\psi\|_{L_{2}(0,1)}\left\|z_{\delta}^{\prime}\right\|_{L_{2}(0,1)}
$$

Hence (2.12) with $M_{1}=1 / K_{1}$.

b) For $m \geq 0$ the proof is analogous, by induction: on differentiating (2.11) $m+1$ times and taking the $L_{2}(0,1)$ inner product of the resulting equality with $z^{(m+2)}$, after a simple manipulation based on integration by parts we obtain the inequality (2.13) where

$$
M_{m+2}=\frac{N_{m+2}}{K_{m+2}}\left[1+\sum_{k=2}^{m+2}\left(\begin{array}{c}
m+2 \\
k
\end{array}\right) L_{k} M_{m+3-k}\right]
$$

and $N_{m+2}$ is the constant in the following Poincaré inequality:

$$
\|\psi\|_{H^{m+1}(0,1)} \leq N_{m+2}|\psi|_{H^{m+1}(0,1)} .
$$

Now we are ready to embark on the a posteriori error analysis of the streamline diffusion finite element method, using the dual problem (2.8). We state the following analogue of Lemma 1. 
Lemma 5 Suppose that $0 \leq s \leq p$ and let $\delta \in W_{\infty}^{s+1}(0,1)$; then,

$$
\left|\left(u-u_{h}, \psi\right)\right| \leq C_{i} \sum_{j=1}^{N}\left(\left\|h r_{h}\right\|_{L_{2}\left(I_{j}\right)}+\left\|\delta r_{h}\right\|_{L_{2}\left(I_{j}\right)}\right) \min _{0 \leq \sigma \leq s}\left\{h_{j}^{\sigma}\left|z_{\delta}\right|_{H^{\sigma+1}\left(I_{j}\right)}\right\},
$$

where $C_{i}$ is the constant from (2.2).

Proof: By the definition of the dual problem (2.8), with $w=u-u_{h}$, and the Galerkin property (2.4), we have that

$$
\begin{aligned}
\left(u-u_{h}, \psi\right) & =B_{\delta}\left(u-u_{h}, z_{\delta}\right)=B_{\delta}\left(u-u_{h}, z_{\delta}-z_{\delta, h}\right) \\
& =\left(r_{h}, z_{\delta}-z_{\delta, h}\right)+\left(r_{h}, \delta\left(z_{\delta}^{\prime}-z_{\delta, h}^{\prime}\right)\right)+r_{h}^{-}\left(z_{\delta}(0)-z_{\delta, h}(0)\right) \equiv \mathrm{I}+\mathrm{II}+\mathrm{III} .
\end{aligned}
$$

For $s$ fixed, $0 \leq s \leq p$, we choose $z_{\delta, h}=P_{h} z_{\delta}$ in I, II and III and proceed in the same way as in the proof of Lemma 1 , to arrive at the desired bound.

We note in passing that when $\delta$ is a constant function on $[0,1]$ the hypotheses of Lemma 4 are satisfied with $K_{1}=K_{2}=\ldots=K_{m+2}=1$. Tracing the constants in the proof, we then find that $M_{1}=M_{2}=\ldots=M_{m+2}=1$. In general, $\delta$ will not be constant on [0,1], so the assumptions on $\delta$ stated in a) and b) of Lemma 4 can be seen as conditions on the variation of the computational mesh, given that in practice $\delta$ is related to $h$. Let us suppose, for example, that $\delta$ is defined as a piecewise linear function whose value at the midpoint of $I_{j}$ is equal to $h_{j}$, the length of the interval $I_{j}$. The hypothesis on $\delta$ from part a) is then satisfied on any mesh which results from a coarse background mesh through successive local bisections, so it does not represent a practical limitation from the point of view of our adaptive mesh refinement algorithm. The conditions which occur in part b) are increasingly more demanding; for large values of $m$ they require that the variation (more precisely, the compression from left to right) of the mesh is small. However, we expect that the mesh-size distribution and hence $\delta$ will approach a piecewise smooth function in the process of mesh adaptation. The potential loss of regularity in $\delta$ and the consequential loss of smoothness in $z_{\delta}$ in some interval $I_{j}$ is compensated by the presence of the factor $h_{j}^{\sigma}$ in (2.15). Indeed, the estimate (2.15) is at least as sharp as the one of Lemma 1. We shall return to this question again in Section 3 in the error analysis of the multi-dimensional problem.

The a posteriori error bound resulting from Lemma 5 is stated below in Corollary 6 . First we introduce some notation. For $k$ a positive integer, we put

$$
\dot{H}^{k}(0,1)=\left\{v \in H^{k}(0,1): v(0)=\ldots=v^{(k-1)}(0)=0\right\} .
$$

For $k=0$, by definition, $\dot{H}^{0}(0,1):=L_{2}(0,1)$. Further, we introduce the negative Sobolev norm $\|\cdot\|_{H^{-k}(0,1)}$ by

$$
\|w\|_{H^{-k}(0,1)}=\sup _{v \in \dot{H}^{k}(0,1)} \frac{|(w, v)|}{|v|_{H^{k}(0,1)}} .
$$

In particular, $\|w\|_{H^{0}(0,1)}=\|w\|_{L_{2}(0,1)}$. On noting that $C^{\infty}[0,1]$ is dense in $L_{2}(0,1)=$ $\dot{H}^{0}(0,1)$ and that the set $\left\{\psi \in C^{\infty}[0,1]: \psi(0)=\ldots=\psi^{(k-1)}(0)=0\right\}$ is dense in $\dot{H}^{k}(0,1)$, $k \geq 1$, we deduce from Lemmas 4 and 5 the following a posteriori error bound for the streamline diffusion method. 
Corollary 6 Suppose that $0 \leq s \leq p$ and let the conditions of Lemma 4 hold with $m=s-1$ (when $s=0$, the conditions in part a) of Lemma 4 are assumed); then the following a posteriori error bound holds:

$$
\left\|u-u_{h}\right\|_{H^{-s}(0,1)} \leq C_{i} M_{s+1}\left(\left\|h^{s+1} r_{h}\right\|_{L_{2}(0,1)}+\left\|h^{s} \delta r_{h}\right\|_{L_{2}(0,1)}\right) .
$$

Unlike the a posteriori error bound discussed in the previous section, this estimate now reflects completely the approximation properties of the test space, with the practically realistic value of $\delta=C_{\delta} h$.

\section{The multi-dimensional model problem}

We consider the hyperbolic boundary-value problem

$$
\mathcal{L} u \equiv \beta \cdot \nabla u+c u=f \quad \text { in } \Omega, \quad u=g \quad \text { on } \Gamma_{-},
$$

where $\Omega=(0,1)^{n}$, with inflow boundary $\Gamma_{-}=\{x \in \partial \Omega: \beta(x) \cdot \nu(x)<0\}$; here $\nu(x)$ denotes the unit outward normal vector at $x \in \partial \Omega$ (defined almost everywhere on $\partial \Omega$ ). Analogously, we define the outflow boundary $\Gamma_{+}=\{x \in \partial \Omega: \beta(x) \cdot \nu(x) \geq 0\}$.

We shall suppose that the entries $\beta_{1}, \ldots, \beta_{n}$ of the $n$-component vector function $\beta$ are continuously differentiable and positive on $\bar{\Omega}$; this ensures that $\partial \Omega$ is non-characteristic for $\mathcal{L}$ at almost every point $x$ on $\partial \Omega$. It will be assumed that $c$ is a continuous real-valued function on $\bar{\Omega}, f$ is a real-valued square-integrable function on $\Omega$, and $g$ is a real-valued square-integrable function on $\Gamma_{-}$.

In order to set up the variational formulation of (3.1), we associate with $\mathcal{L}$ the function space

$$
H(\Omega)=\left\{v \in L_{2}(\Omega): \mathcal{L} v \in L_{2}(\Omega)\right\}
$$

we consider the bilinear form $B(\cdot, \cdot): H(\Omega) \times H(\Omega) \rightarrow \mathbf{R}$ defined by

$$
B(w, v)=(\mathcal{L} w, v)-((\beta \cdot \nu) w, v)_{\Gamma_{-}}
$$

and introduce the linear form $l: H(\Omega) \rightarrow \mathbf{R}$ by

$$
l(v)=(f, v)-((\beta \cdot \nu) g, v)_{\Gamma_{-}} .
$$

In these definitions $(\cdot, \cdot)$ denotes the $L_{2}$ inner product over $\Omega$ and $(\cdot, \cdot)_{\Gamma_{-}}$signifies the $L_{2}$ inner product over $\Gamma_{-}$(with $(\cdot, \cdot)_{\Gamma_{+}}$being defined analogously). It can be shown that any function $v \in H(\Omega)$ has a well defined trace on $\Gamma_{-}$(respectively, $\left.\Gamma_{+}\right)$in $L_{2}\left(\Gamma_{-}\right)$(respectively, $\left.L_{2}\left(\Gamma_{+}\right)\right)$, so the definitions of $B(\cdot, \cdot)$ and $l(\cdot)$ are meaningful. When equipped with the graph-norm

$$
\|v\|_{H(\Omega)}=\left(\|v\|_{L_{2}(\Omega)}^{2}+\|\mathcal{L} v\|_{L_{2}(\Omega)}^{2}\right)^{1 / 2},
$$

$H(\Omega)$ is a Hilbert space. With this notation, the boundary-value problem (3.1) can be expressed as follows: find $u$ in $H(\Omega)$ such that

$$
B(u, v)=l(v) \quad \forall v \in H(\Omega) .
$$


Using the Lax-Milgram Theorem, it is a straightforward matter to prove that under the present hypotheses on $\beta, c, f$ and $g$ problem (3.2) has a unique solution $u$ in $H(\Omega)$.

Let $\mathcal{T}_{h}=\{\kappa\}$ be a finite element partition of $\Omega$ into open element domains $\kappa$. We shall suppose that the family of partitions $\left\{\mathcal{T}_{h}\right\}$ is shape regular; namely, there exists a positive constant $\alpha$ such that

$$
\frac{h_{\kappa}}{\rho_{\kappa}} \leq \alpha \quad \forall \kappa \in \bigcup_{h} \mathcal{T}_{h}
$$

with $h_{\kappa}=\operatorname{diam}(\bar{\kappa})$ and $\rho_{\kappa}$ the diameter of the largest ball contained in $\bar{\kappa}$. Let $h(x)$ denote the mesh function whose value on element $\kappa$ is equal to $h_{\kappa}$. On $\mathcal{T}_{h}$ we consider the finite element space $S_{h} \subset H^{1}(\Omega)(\subset H(\Omega))$ containing continuous piecewise polynomials of maximum degree $p, p \geq 1$. It will be assumed that $S_{h}$ possesses the following standard approximation property:

(B) Given that $v \in H^{s+1}(\Omega)$ and $\left.v\right|_{\Gamma_{-}} \in H^{s+1}\left(\Gamma_{-}\right)$for some $s, 0 \leq s \leq p$, there exists $v_{h}$ in $S_{h}$ and a constant $C_{i}$, independent of $v$ and the mesh function $h$, such that

$$
\begin{aligned}
& \left\|v-v_{h}\right\|_{L_{2}(\kappa)}+h_{\kappa}\left|v-v_{h}\right|_{H^{1}(\kappa)} \leq C_{i} h_{\kappa}^{s+1}|v|_{H^{s+1}(\hat{\kappa})} \quad \forall \kappa \in \mathcal{T}_{h}, \\
& \left\|v-v_{h}\right\|_{L_{2}\left(\partial \kappa \cap \Gamma_{-}\right)} \leq C_{i} h_{\kappa}^{s+1}|v|_{H^{s+1}\left(\partial \hat{\kappa} \cap \Gamma_{-}\right)} \quad \forall \kappa \in \mathcal{T}_{h} \text { s.t. } \partial \kappa \cap \Gamma_{-} \neq \emptyset .
\end{aligned}
$$

Here and in the rest of the paper, for $s \leq n / 2, \hat{\kappa}$ signifies the union of all such elements whose closure has nonempty intersection with the closure of $\kappa$; for $s>n / 2$, we define $\hat{\kappa}=\kappa$. Given a function $v$ and an associated function $v_{h}$ satisfying (3.3), (3.4), we shall write $v_{h}=P_{h} v$ to denote that $v_{h}$ is assigned to $v$.

Remark 7 For $s>n / 2$ hypothesis $(B)$ can be satisfied by selecting $P_{h} v$ as the finite element interpolant of $v \in H^{s+1}(\Omega)$ from $S_{h}$. For $s \leq n / 2$, point evaluation of $v$ need not be meaningful, due to the lack of Sobolev regularity; in this case $P_{h} v$ can be chosen as a suitable quasi-interpolant of $v$. A further possibility is to define $P_{h} v \in S_{h}$ at degrees of freedom interior to $\Omega \cup \Gamma_{+}$as indicated in the previous sentence, while on $\Gamma_{-}$one can define $\left.P_{h} v\right|_{\Gamma_{-}}$as the orthogonal projection of $\left.v\right|_{\Gamma_{-}}$onto the restriction of $S_{h}$ to $\Gamma_{-}$with respect to the inner product

$$
\int_{\Gamma_{-}}|\beta \cdot \nu| w(x) v(x) \mathrm{ds} .
$$

This latter choice of $P_{h}$ will be made use of in Remark 17 below.

Next, we introduce the finite element approximation of (3.2); to do so, we consider the stabilisation parameter as a positive function $\delta$ contained in $L_{\infty}(\Omega)$, the bilinear form $B_{\delta}(\cdot, \cdot)$ defined on $H(\Omega) \times H(\Omega)$ by

$$
B_{\delta}(w, v)=(\mathcal{L} w, v+\delta \hat{\mathcal{L}} v)-((\beta \cdot \nu) w, v)_{\Gamma_{-}},
$$

where, following Baiocchi and Brezzi [1], we put $\hat{\mathcal{L}} w=\beta \cdot \nabla w+\hat{c} w$ with

$$
\hat{c}= \begin{cases}0 & \text { for the streamline diffusion finite element method, } \\ c & \text { for the least-squares stabilised finite element method, } \\ \nabla \cdot \beta-c & \text { for the negative-adjoint stabilised finite element method, }\end{cases}
$$


and introduce the linear functional $l_{\delta}(\cdot): H(\Omega) \rightarrow \mathbf{R}$ by

$$
l_{\delta}(v)=(f, v+\delta \hat{\mathcal{L}} v)-((\beta \cdot \nu) g, v)_{\Gamma_{-}} .
$$

With these notations, we formulate the stabilised Galerkin finite element method: find $u_{h} \in S_{h}$ such that

$$
B_{\delta}\left(u_{h}, v_{h}\right)=l_{\delta}\left(v_{h}\right) \quad \forall v_{h} \in S_{h} .
$$

Remark 8 In the case of the streamline diffusion method and the least-square stabilised finite element method, assuming, for example, that there exists a nonnegative constant $c_{0}$ such that

$$
c-\frac{1}{2} \nabla \cdot \beta \geq c_{0} \quad \text { on } \bar{\Omega},
$$

it is a straightforward matter to prove that $B_{\delta}\left(v_{h}, v_{h}\right)>0$ for all $v_{h}$ in $S_{h} \backslash\{0\}$. The same is true in the case of the negative-adjoint stabilised finite element method, provided that (3.7) holds with $c_{0}>0$ and $\delta \leq \frac{1}{2} c_{0}\left[c^{2}+(\nabla \cdot \beta)^{2}\right]^{-1}$ on $\bar{\Omega}$. As $S_{h}$ is finite-dimensional, it follows for each of the three choices of $\hat{\mathcal{L}}$ above that (3.6) has a unique solution $u_{h}$ in $S_{h}$.

\subsection{A posteriori error analysis for norms}

The a posteriori error analysis of (3.6) is based on considering the finite element residual and the boundary residual defined, respectively, by

$$
r_{h}=f-\mathcal{L} u_{h} \quad \text { and } \quad r_{h}^{-}=\left.\left(g-u_{h}\right)\right|_{\Gamma_{-}},
$$

and noting the Galerkin property

$$
B_{\delta}\left(u-u_{h}, v_{h}\right)=0 \quad \forall v_{h} \in S_{h} .
$$

As in the second approach in one-dimension, we proceed by introducing the stabilisationdependent dual problem: find $z_{\delta} \in H(\Omega)$ such that

$$
B_{\delta}\left(w, z_{\delta}\right)=(w, \psi) \quad \forall w \in H(\Omega),
$$

where $\psi$ is a given function in $C_{0}^{\infty}(\Omega)$. The existence of a unique solution $z_{\delta}$ in $H(\Omega)$ to $(3.9)$ follows by applying the Lax-Milgram Theorem; see also Theorem 1.4.1 in the monograph of Oleinik and Radkevič [11]. For the moment we shall suppose that $z_{\delta}$ is sufficiently smooth, namely $z_{\delta} \in H^{s+1}(\Omega), 0 \leq s \leq p$; conditions which ensure this will be stated below in Lemma 10. We begin with the following preliminary result.

Lemma 9 Suppose that $0 \leq s \leq p$ and $z_{\delta} \in H^{s+1}(\Omega)$; then,

$$
\begin{aligned}
\left|\left(u-u_{h}, \psi\right)\right| \leq & C_{i} N_{1} \sum_{e: e \cap \Gamma_{-} \neq \emptyset}\left\|h_{e}^{1 / 2} r_{h}^{-}\right\|_{L_{2}(e)} \min _{0 \leq \sigma \leq s}\left\{h_{e}^{\sigma}\left|z_{\delta}\right|_{H^{\sigma+1}\left(\hat{\kappa}_{e}\right)}\right\} \\
& +C_{i} \sum_{\kappa \in \mathcal{T}_{h}}\left(\left\|\left.h_{\kappa} r_{h}\right|_{L_{2}(\kappa)}+N_{2}\right\| \delta r_{h} \|_{L_{2}(\kappa)}\right) \min _{0 \leq \sigma \leq s}\left\{h_{\kappa}^{\sigma}\left|z_{\delta}\right|_{H^{\sigma+1}(\hat{\kappa})}\right\},
\end{aligned}
$$

where $N_{1}=\sqrt{2 C_{3}}\|\beta\|_{L_{\infty}\left(\Gamma_{-}\right)}, N_{2}=\max _{\kappa \in \mathcal{T}_{h}}\left(\|\beta\|_{L_{\infty}(\kappa)}+h_{\kappa}\|\hat{c}\|_{L_{\infty}(\kappa)}\right), C_{i}$ is the constant from condition (B), $C_{3}$ is a positive constant that depends only on the mesh-regularity parameter $\alpha$ and the number of space dimensions $n, \kappa_{e}$ is the element with (open) face $e$ and $h_{e}$ is the diameter of $e$. 
Proof: Choosing $w=u-u_{h}$ in (3.9) and noting the Galerkin property, we deduce that

$$
\begin{aligned}
& \left(u-u_{h}, \psi\right)=B_{\delta}\left(u-u_{h}, z_{\delta}\right)=B_{\delta}\left(u-u_{h}, z_{\delta}-z_{\delta, h}\right) \\
& \quad=\left(r_{h}, z_{\delta}-z_{\delta, h}\right)+\left(r_{h}, \delta \hat{\mathcal{L}}\left(z_{\delta}-z_{\delta, h}\right)\right)-\left((\beta \cdot \nu) r_{h}^{-},\left(z_{\delta}-z_{\delta, h}\right)\right)_{\Gamma_{-}} \equiv \mathrm{I}+\mathrm{II}+\mathrm{III},
\end{aligned}
$$

where $z_{\delta, h}=P_{h} z_{\delta}$ (cf. (B) above). We shall proceed by estimating each of the terms I, II and III by means of the approximation property (B). First, for any $\sigma_{\kappa} \in[0, s]$, we have

$$
|\mathrm{I}| \leq C_{i} \sum_{\kappa \in \mathcal{T}_{h}}\left\|h_{\kappa} r_{h}\right\|_{L_{2}(\kappa)} h_{\kappa}^{\sigma_{\kappa}}\left|z_{\delta}\right|_{H^{\sigma_{\kappa}+1}(\hat{\kappa})}
$$

and therefore,

$$
|\mathrm{I}| \leq C_{i} \sum_{\kappa \in \mathcal{T}_{h}}\left\|h_{\kappa} r_{h}\right\|_{L_{2}(\kappa)} \min _{0 \leq \sigma \leq s}\left\{h_{\kappa}^{\sigma}\left|z_{\delta}\right|_{H^{\sigma+1}(\hat{\kappa})}\right\}
$$

Second,

$$
|\mathrm{II}| \leq C_{i} \sum_{\kappa \in \mathcal{T}_{h}}\left(\|\beta\|_{L_{\infty}(\kappa)}+h_{\kappa}\|\hat{c}\|_{L_{\infty}(\kappa)}\right)\left\|\delta r_{h}\right\|_{L_{2}(\kappa)} \min _{0 \leq \sigma \leq s}\left\{h_{\kappa}^{\sigma}\left|z_{\delta}\right|_{H^{\sigma+1}(\hat{\kappa})}\right\}
$$

Third, denoting by $e$ an open face of an element in the partition $\mathcal{T}_{h}$, we deduce that

$$
|\mathrm{III}| \leq \sum_{e: e \cap \Gamma_{-} \neq \emptyset}\|\beta\|_{L_{\infty}(e)}\left\|r_{h}^{-}\right\|_{L_{2}(e)}\left\|z_{\delta}-z_{\delta, h}\right\|_{L_{2}(e)} .
$$

To each open face $e$ which has nonempty intersection with $\Gamma_{-}$we assign the element $\kappa_{e}$ in $\mathcal{T}_{h}$ with that face. Now,

$$
\left\|z_{\delta}-z_{\delta, h}\right\|_{L_{2}(e)}^{2} \leq C_{3}\left(\left\|z_{\delta}-z_{\delta, h}\right\|_{L_{2}\left(\kappa_{e}\right)}\left|z_{\delta}-z_{\delta, h}\right|_{H^{1}\left(\kappa_{e}\right)}+\frac{1}{h_{\kappa_{e}}}\left\|z_{\delta}-z_{\delta, h}\right\|_{L_{2}\left(\kappa_{e}\right)}^{2}\right),
$$

where $C_{3}$ is a positive constant which only depends on the mesh-regularity parameter $\alpha$ and the number of space dimensions, $n$. Consequently, by (B),

$$
\| z_{\delta}-\left.z_{\delta, h}\right|_{L_{2}(e)} \leq C_{i} \sqrt{2 C_{3}} h_{\kappa_{e}}^{s+1 / 2}\left|z_{\delta}\right|_{H^{s+1}\left(\hat{\kappa}_{e}\right)} .
$$

Inserting this into the bound on term III yields, possibly with an altered value of $C_{3}=$ $C_{3}(\alpha, n)$,

$$
|\mathrm{III}| \leq C_{i} \sqrt{2 C_{3}} \sum_{e: e \cap \Gamma_{-} \neq \emptyset}\|\beta\|_{L_{\infty}(e)}\left\|h_{e}^{1 / 2} r_{h}^{-}\right\|_{L_{2}(e)} \min _{0 \leq \sigma \leq s}\left\{h_{e}^{\sigma}\left|z_{\delta}\right|_{H^{\sigma+1}\left(\hat{\kappa}_{e}\right)}\right\}
$$

Upon substituting the bounds on I, II and III into (3.11), we obtain (3.10).

Now we shall verify that $z_{\delta} \in H^{s+1}(\Omega)$ and derive a bound on $\left|z_{\delta}\right|_{H^{s+1}(\Omega)}$ in terms of the $H^{s+1}(\Omega)$ norm of $\psi$.

Lemma 10 Suppose that $s \geq 0, \beta \in\left[C^{s+2}(\bar{\Omega})\right]^{n}, c \in C^{s+1}(\bar{\Omega}), \delta \in W_{\infty}^{s+1}(\Omega), \psi \in C_{0}^{\infty}(\Omega)$, and assume that there exists positive constants $L_{s+1}$ and $K_{s+1}$ such that

$$
\max _{|\alpha| \leq s+1}\left|D^{\alpha} \delta(x)\right| \leq L_{s+1}, \quad 1-(s+1)|\nabla \delta(x)|_{1}|\beta(x)|_{1} \geq K_{s+1} \quad \forall x \in \Omega,
$$


where $|\cdot|_{1}$ denotes the $l_{1}$ vector norm on $\mathbf{R}^{n}$. Let us also assume that $\nabla \cdot \beta-c-\hat{c} \equiv 0$ in a small but fixed neighbourhood of the origin, independent of $\delta$; then, there exists a constant $M_{s+1}=M_{s+1}\left(K_{s+1}, L_{s+1}\right)$, independent of $\delta$ and $\psi$, such that

$$
\left\|z_{\delta}\right\|_{H^{s+1}(\Omega)} \leq M_{s+1}\|\psi\|_{H^{s+1}(\Omega)} .
$$

We note here that $\nabla \cdot \beta-c-\hat{c} \equiv 0$ is a compatibility condition between the data of the partial differential equation and the homogeneous inflow boundary datum at the vertex point of the inflow boundary.

Proof: Since $\delta \in W_{\infty}^{1}(\Omega)$, (3.9) can be rewritten in the following strong form:

$$
\begin{aligned}
& -\nabla \cdot\left(\beta\left(z_{\delta}+\delta\left(\beta \cdot \nabla z_{\delta}+\hat{c} z_{\delta}\right)\right)+c\left(z_{\delta}+\delta\left(\beta \cdot \nabla z_{\delta}+\hat{c} z_{\delta}\right)\right)=\psi \text { in } \Omega\right. \\
& \beta \cdot \nabla z_{\delta}+\hat{c} z_{\delta}=0 \text { on } \Gamma_{-}, \\
& z_{\delta}+\delta\left(\beta \cdot \nabla z_{\delta}+\hat{c} z_{\delta}\right)=0 \text { on } \Gamma_{+} \text {. }
\end{aligned}
$$

Putting $\chi=z_{\delta}+\delta\left(\beta \cdot \nabla z_{\delta}+\hat{c} z_{\delta}\right)$, we can write (3.14) as a system of coupled scalar hyperbolic equations:

$$
\begin{aligned}
& -\nabla \cdot(\beta \chi)+c \chi=\psi \text { in } \Omega, \quad \chi=0 \quad \text { on } \Gamma_{+}, \\
& z_{\delta}+\delta\left(\beta \cdot \nabla z_{\delta}+\hat{c} z_{\delta}\right)=\chi \quad \text { on } \Omega, \quad z_{\delta}=\chi \quad \text { on } \Gamma_{-} \text {. }
\end{aligned}
$$

First we shall bound $\chi$ in terms of $\psi$, then $z_{\delta}$ in terms of $\chi$; combining the two will yield the desired bound on $z_{\delta}$ in terms of $\psi$.

Recalling that $\psi \in C_{0}^{\infty}(\Omega)$, it follows by the Differentiability Theorem of Rauch ([14], p. 272) applied to (3.15) that

$$
\|\chi\|_{H^{s+1}(\Omega)} \leq M_{s+1}\|\psi\|_{H^{s+1}(\Omega)},
$$

where $M_{s+1}$ is a positive constant, independent of $\psi$ (and of $\delta$, of course).

Next we shall derive bounds on Sobolev norms of $z_{\delta}$ in terms of $\chi$, independent of the stabilisation parameter $\delta$. We begin by reformulating (3.16) so that the boundary condition is homogeneous: we put $w=z_{\delta}-\chi$ and observe that $w$ is the solution of the following boundary value problem

$$
\begin{aligned}
\beta \cdot \nabla w+\left(\hat{c}+\frac{1}{\delta}\right) w & =-(\beta \cdot \nabla \chi+\hat{c} \chi) & & \text { in } \Omega \\
w & =0 & & \text { on } \Gamma_{-} .
\end{aligned}
$$

Further, denoting the right-hand side in (3.18) by $\phi$, we see from (3.15) that

$$
\phi \equiv-(\beta \cdot \nabla \chi+\hat{c} \chi)=\psi+\chi(\nabla \cdot \beta-c-\hat{c}) .
$$

With this new notation (3.18), (3.19) becomes

$$
\begin{aligned}
\delta \beta \cdot \nabla w+(1+\delta \hat{c}) w & =\delta \phi & \text { in } \Omega, \\
w & =0 & \text { on } \Gamma_{-} .
\end{aligned}
$$


Since $\psi \in C_{0}^{\infty}(\Omega)$ and $\nabla \cdot \beta-c-\hat{c}$ vanishes in the vicinity of the origin, it follows that $\phi \in H^{s+1}(\Omega)$ and $\phi$ vanishes in the vicinity of the origin. Thus, from Rauch's theorem, we deduce that $w \in H^{s+1}(\Omega)$ and

$$
\|w\|_{H^{s+1}(\Omega)} \leq M_{s+1}\|\phi\|_{H^{s+1}(\Omega)},
$$

where $M_{s+1}$ is a positive constant (possibly different than in (3.17)). The independence of $M_{s+1}$ on $\delta$ follows by tracing the constants in Rauch's proof, making use of (3.12). Finally, we recall that $z_{\delta}=\chi+w$ and apply the bounds on $\chi$ and $w$.

Now we are ready to complete the a posteriori error analysis we embarked on before the statement of Lemma 10. In particular, we derive a posteriori bounds on negative Sobolev norms of the global error $u-u_{h}$. Given that $m$ is a positive integer, we define $H_{0}^{m}(\Omega)$ as the closure of $C_{0}^{\infty}(\Omega)$ in the norm of $H^{m}(\Omega)$ and introduce the negative Sobolev norm $\|\cdot\|_{H^{-m}(\Omega)}$ in the usual way by

$$
\|w\|_{H^{-m}(\Omega)}=\sup _{v \in C_{0}^{\infty}(\Omega)} \frac{|(w, v)|}{\|v\|_{H^{m}(\Omega)}} .
$$

The next result follows by applying (3.13) to the right-hand side of (3.10).

Theorem 11 Suppose that $0 \leq s \leq p$ and assume that the conditions of Lemma 10 hold; then, we have the following a posteriori error bound:

$$
\begin{aligned}
\left\|u-u_{h}\right\|_{H^{-s-1}(\Omega)} \leq & M_{s+1}\left\{C_{i}\left(\sum_{\kappa \in \mathcal{T}_{h}}\left\|h_{\kappa}^{s+1} r_{h}\right\|_{L_{2}(\kappa)}^{2}+N_{2}^{2}\left\|h_{\kappa}^{s} \delta r_{h}\right\|_{L_{2}(\kappa)}^{2}\right)^{1 / 2}\right. \\
& \left.+C_{i} N_{1}\left(\sum_{e: e \cap \Gamma_{-} \neq \emptyset}\left\|h_{e}^{s+1 / 2} r_{h}^{-}\right\|_{L_{2}(e)}^{2}\right)^{1 / 2}\right\}
\end{aligned}
$$

where $M_{s+1}$ is a positive constant, independent of $\delta, C_{i}$ is the constant from condition (B), and $N_{1}$ and $N_{2}$ are as in Lemma 9.

\subsection{A posteriori error estimation for linear functionals}

In many problems of physical importance the quantity of interest is a linear functional of the solution. Relevant examples include the lift and drag coefficients for a body immersed into an inviscid fluid, the local mean value of the field, or its flux through the outflow boundary of the computational domain.

Suppose that we wish to control the discretisation error in terms of some linear functional $J(\cdot)$ defined on the space $H(\Omega)$ (or on a suitable subspace which contains the finite element space $S_{h}$ and the exact solution $u$ of problem (3.1)). To do so, following the same line of thought as in the one-dimensional case, we shall derive an a posteriori bound on the error between $J(u)$ and $J\left(u_{h}\right)$, where $u_{h} \in S_{h}$ is the finite element approximation to $u$, defined by (3.6). The error analyses which we shall perform are based on two dual problems, 
the first of which stems from the formal adjoint of the partial differential operator while the second exploits the adjoint of the stabilised primal operator:

$$
\text { Find } z \text { in } H(\Omega) \text { such that } \quad B(w, z)=J(w) \quad \forall w \in H(\Omega) \text {; }
$$

and

$$
\text { Find } z_{\delta} \text { in } H(\Omega) \text { such that } \quad B_{\delta}\left(w, z_{\delta}\right)=J(w) \quad \forall w \in H(\Omega) .
$$

Let us assume that each of these problems possesses a unique solution. Clearly, the validity of this assumption depends on the choice of the linear functional $J(\cdot)$. We have the following general result.

Theorem 12 The dual problems (3.24) and (3.25) give rise to the following error representation formulas, respectively:

$$
\begin{aligned}
& J(u)-J\left(u_{h}\right)=-\left((\beta \cdot \nu) r_{h}^{-},\left(z-z_{h}\right)\right)_{\Gamma_{-}}+\left(r_{h}, z-z_{h}\right)-\left(r_{h}, \delta \hat{\mathcal{L}}_{z_{h}}\right), \\
& J(u)-J\left(u_{h}\right)=-\left((\beta \cdot \nu) r_{h}^{-},\left(z_{\delta}-z_{\delta, h}\right)\right)_{\Gamma_{-}}+\left(r_{h}, z_{\delta}-z_{\delta, h}\right)+\left(r_{h}, \delta \hat{\mathcal{L}}\left(z_{\delta}-z_{\delta, h}\right)\right) .
\end{aligned}
$$

ProOF: We shall only present the argument in the case of the stabilisation-dependent dual problem (3.25); for the standard dual problem (3.24) the proof is analogous. On choosing $w=u-u_{h}$ in (3.25) and recalling the Galerkin property (3.8), we deduce that

$$
\begin{aligned}
J(u)-J\left(u_{h}\right) & =J\left(u-u_{h}\right)=B_{\delta}\left(u-u_{h}, z_{\delta}\right)=B_{\delta}\left(u-u_{h}, z_{\delta}-z_{\delta, h}\right) \\
& =-\left((\beta \cdot \nu) r_{h}^{-}, z_{\delta}-z_{\delta, h}\right)_{\Gamma_{-}}+\left(r_{h}, z_{\delta}-z_{\delta, h}\right)+\left(\delta r_{h}, \hat{\mathcal{L}}\left(z_{\delta}-z_{\delta, h}\right)\right) .
\end{aligned}
$$

That completes the proof of $(3.26 \mathrm{~b})$.

Guided by the error representation formulas (3.26a) and (3.26b), we define the residual terms $\rho^{(i)}, i=1,2,3$, by

$$
\rho^{(1)}=-(\beta \cdot \nu) r_{h}^{-}, \quad \rho^{(2)}=\rho^{(3)}=r_{h},
$$

and the weighting terms $\omega^{(i)}, i=1,2,3$, as follows:

a) for dual problem (3.24):

$$
\omega^{(1)}=\omega^{(2)}=z-z_{h}, \quad \omega^{(3)}=-\delta \hat{\mathcal{L}} z_{h} ;
$$

b) for the stabilisation-dependent dual problem (3.25):

$$
\omega^{(1)}=\omega^{(2)}=z_{\delta}-z_{\delta, h}, \quad \omega^{(3)}=\delta \hat{\mathcal{L}}\left(z_{\delta}-z_{\delta, h}\right),
$$

for $z_{h}$ and $z_{\delta, h}$ in $S_{h}$. Using this notation, (3.26a) and (3.26b) can be rewritten in the following compact form:

$$
J(u)-J\left(u_{h}\right)=\left(\rho^{(1)}, \omega^{(1)}\right)_{\Gamma_{-}}+\left(\rho^{(2)}, \omega^{(2)}\right)+\left(\rho^{(3)}, \omega^{(3)}\right) .
$$

Given a linear functional $J(\cdot)$ and a positive tolerance $T O L$, the aim of the computation is to calculate $u_{h}$ so that

$$
\left|J(u)-J\left(u_{h}\right)\right| \leq T O L .
$$


A sufficient condition for this to hold is that the stopping criterion

$$
\mathcal{E}_{1}\left(u_{h}\right) \leq T O L
$$

is satisfied, where

$$
\mathcal{E}_{1}\left(u_{h}\right) \equiv\left|\left(\rho^{(1)}, \omega^{(1)}\right)_{\Gamma_{-}}+\left(\rho^{(2)}, \omega^{(2)}\right)+\left(\rho^{(3)}, \omega^{(3)}\right)\right| .
$$

If (3.28) holds true, $J\left(u_{h}\right)$ is accepted as an accurate representation of $J(u)$; otherwise, $u_{h}$ is discarded and a new approximation is computed on a finer partition. In order to ensure that the partition is refined only where necessary, a local decision has to be made on each element $\kappa$ as to whether $\operatorname{diam}(\kappa)$ is acceptable in relation to TOL. A convenient approach to constructing a refinement criterion which relates the local element size to $T O L$ is to localise the right-hand side in (3.29). More precisely, $\mathcal{E}_{1}\left(u_{h}\right)$ is further bounded from above by decomposing the inner products over $\Omega$ and $\Gamma_{-}$as sums of inner products over elements $\kappa$ in $\Omega$ and faces $\partial \kappa$ in $\Gamma_{-}$, respectively, with the absolute value sign now appearing under the summation sign:

$$
\left|J(u)-J\left(u_{h}\right)\right| \leq \mathcal{E}_{2}\left(u_{h}\right),
$$

where

$$
\mathcal{E}_{2}\left(u_{h}\right) \equiv \sum_{\kappa \in \mathcal{T}_{h}}\left|\left(\rho_{\kappa}^{(1)}, \omega_{\kappa}^{(1)}\right)_{\partial \kappa \cap \Gamma_{-}}+\left(\rho_{\kappa}^{(2)}, \omega_{\kappa}^{(2)}\right)_{\kappa}+\left(\rho_{\kappa}^{(3)}, \omega_{\kappa}^{(3)}\right)_{\kappa}\right|
$$

with

$$
\rho_{\kappa}^{(i)}=\left.\rho^{(i)}\right|_{\kappa}, \quad \omega_{\kappa}^{(i)}=\left.\omega^{(i)}\right|_{\kappa}, \quad i=1,2,3 \quad \forall \kappa \in \mathcal{T}_{h} .
$$

Thus, for example, a possible refinement criterion might consist of checking whether on each element $\kappa$ in the partition $\mathcal{T}_{h}$ the following inequality holds:

$$
\left|\left(\rho_{\kappa}^{(1)}, \omega_{\kappa}^{(1)}\right)_{\partial \kappa \cap \Gamma_{-}}+\left(\rho_{\kappa}^{(2)}, \omega_{\kappa}^{(2)}\right)_{\kappa}+\left(\rho_{\kappa}^{(3)}, \omega_{\kappa}^{(3)}\right)_{\kappa}\right| \leq \frac{T O L}{N}
$$

where $N$ is the number of elements in $\mathcal{T}_{h}$. If (3.32) fails on an element $\kappa$, then $\kappa$ is subdivided; otherwise $\kappa$ is accepted as being of adequate size, for if (3.32) holds on each element $\kappa$ in $\mathcal{T}_{h}$ then, according to (3.30), the required error control (3.27) has been achieved. We note that other mesh refinement criteria are also plausible (see, for example, [3, 13] and Section 4 below), but these too will typically rely on localising $\mathcal{E}_{1}\left(u_{h}\right)$ in a manner discussed above. As a precursor to the numerical experiments in Section 4 we remark here that the seemingly harmless transition from (3.29) to (3.31) can be detrimental: degradation of the asymptotic properties of the bound and uneconomical meshes may result. In fact, it will be shown in Section 4.4 that the occurrence of such adverse effects (stemming from the localisation of global error bounds) is closely related to the choice of the dual problem.

While the residual terms $\rho_{\kappa}^{(i)}, i=1,2,3$, can be easily evaluated once the numerical solution $u_{h}$ has been computed, the calculation of the weights $\omega_{\kappa}^{(i)}, i=1,2,3$, requires special care; this will be discussed in detail in the next section. First, however, we perform two 'thought experiments' which illuminate the significance of using the stabilisation-dependent dual problem (3.25) and illustrate the implications of our a posteriori error analysis. We 
consider the linear advection equation (3.1) on the unit square $\Omega=(0,1)^{2}$ in the $x y$ coordinate system, with $\beta=(1,1)^{T}, c=0$ and $f=0$. The inflow boundary datum $g$ will be assumed to be such that the exact solution $u$ is smooth, but not piecewise constant or linear.

Thought experiment 1. First, we choose the mean-flow across the outflow boundary $\Gamma_{+}$ as the functional to be controlled; namely, we take

$$
J(w)=(1,(\beta \cdot \nu) w)_{\Gamma_{+}} .
$$

In this case, the solution $z=1$ of the standard dual problem

$$
-\beta \cdot \nabla z=0 \quad \text { in } \Omega, \quad z=1 \quad \text { on } \Gamma_{+},
$$

is also the solution of the corresponding stabilisation-dependent dual problem (3.25). This implies that $J(u)=J\left(u_{h}\right)$, recovering the well known conservation property of the scheme (3.6).

Thought experiment 2. Next, we choose as the functional to be controlled the mean-flow over $\Omega$; thus, we take

$$
J(w)=(1, w) .
$$

The solution of the corresponding standard dual problem

$$
-\beta \cdot \nabla z=1 \quad \text { in } \Omega, \quad z=0 \quad \text { on } \Gamma_{+},
$$

is area-wise linear with an edge along the line $y=x$. This results in nonzero values for the error indicators $\left(r_{h}, \delta\left(\beta \cdot \nabla z_{h}\right)\right)_{\kappa}$ in all of $\Omega$ inducing global mesh refinement which does not seem necessary for this particular functional, in view of the conservation property of the scheme. Now we slightly change the error functional $J(\cdot)$ to

$$
J_{\delta}(w)=(1, w)-(1, \delta(\beta \cdot \nu) w)_{\Gamma_{-}},
$$

and assume that the stabilisation parameter $\delta$ is a constant function over $\Omega$; then, a simple calculation shows that the corresponding stabilisation-dependent dual problem (3.25) can be rewritten as

$$
\left(-\beta \cdot \nabla z_{\delta}, w-\delta(\beta \cdot \nabla w)\right)+\left((\beta \cdot \nu) z_{\delta}, w\right)_{\Gamma_{+}}=(1, w)-\delta(1,(\beta \cdot \nu) w)_{\Gamma_{-}} \quad \forall w \in H(\Omega),
$$

and it has the same area-wise linear solution as the problem

$$
-\beta \cdot \nabla z_{\delta}=1 \quad \text { in } \Omega, \quad z_{\delta}=\delta \quad \text { on } \Gamma_{+} .
$$

Consequently, the local error indicators $\left|\left(\rho_{\kappa}^{(2)}, \omega_{\kappa}^{(2)}\right)_{\kappa}\right|+\left|\left(\rho_{\kappa}^{(3)}, \omega_{\kappa}^{(3)}\right)_{\kappa}\right|$ are nonzero only along the characteristic line $y=x$. This indicates that mesh refinement based on the a posteriori error bound which stems from the stabilisation-dependent dual problem will be localised to the line $y=x$ and will be, therefore, highly economical.

Definition 13 For future reference, we recall the particular forms of the terms on the right-hand sides of (3.26a) and (3.26b), and we define

$$
I_{1} \equiv\left|\left((\beta \cdot \nu) r_{h}^{-}, z-z_{h}\right)_{\Gamma_{-}}\right|, \quad I I_{1} \equiv\left|\left(r_{h}, z-z_{h}\right)\right|, \quad I I I_{1} \equiv\left|\left(\delta r_{h}, \hat{\mathcal{L}} z_{h}\right)\right|
$$


and

$$
I_{1, \delta} \equiv\left|\left((\beta \cdot \nu) r_{h}^{-}, z_{\delta}-z_{\delta, h}\right)_{\Gamma_{-}}\right|, \quad I I_{2, \delta} \equiv\left|\left(r_{h}, z_{\delta}-z_{\delta, h}\right)\right|, \quad I I I_{1, \delta} \equiv \mid\left(\delta r_{h}, \hat{\mathcal{L}}\left(z_{\delta}-z_{\delta, h}\right) \mid .\right.
$$

We also consider the localised counterparts of these, given by

$$
I_{2} \equiv \sum_{\kappa \in \mathcal{T}_{h}}\left|\left((\beta \cdot \nu) r_{h}^{-}, z-z_{h}\right)_{\partial \kappa \cap \Gamma_{-}}\right|, \quad I I_{2} \equiv \sum_{\kappa \in \mathcal{T}_{h}}\left|\left(r_{h}, z-z_{h}\right)_{\kappa}\right|, \quad I I I_{2} \equiv \sum_{\kappa \in \mathcal{T}_{h}}\left|\left(\delta r_{h}, \hat{\mathcal{L}} z_{h}\right)_{\kappa}\right|,
$$

and the corresponding terms $I_{2, \delta}, I I_{2, \delta}, I I I_{2, \delta}$ for the stabilisation-dependent dual problem, arising from the right-hand side of (3.31).

By applying the Cauchy-Schwarz inequality on $\mathrm{I}_{2}, \mathrm{II}_{2}$ and $\mathrm{III}_{2}$, as well as $\mathrm{I}_{2, \delta}, \mathrm{II}_{2, \delta}$ and $\mathrm{III}_{2, \delta}$ together with the approximation property (B), these terms can be further bounded above. Thus we arrive at the following result.

Lemma 14 Let $u$ and $u_{h}$ denote the solutions of (3.1) and (3.6), respectively. Given that $z \in H^{s+1}\left(\Gamma_{-}\right)$for some $s, 0 \leq s \leq p$, we have that

$$
\mathrm{I}_{2} \leq C_{i} \sum_{\kappa \in \mathcal{T}_{h}}\|\beta\|_{L_{\infty}\left(\partial \kappa \cap \Gamma_{-}\right)}\left\|h r_{h}^{-}\right\|_{L_{2}\left(\partial \kappa \cap \Gamma_{-}\right)} \min _{0 \leq \sigma \leq s}\left\{h_{\kappa}^{\sigma}|z|_{H^{\sigma+1}\left(\partial \hat{\kappa} \cap \Gamma_{-}\right)}\right\} .
$$

Furthermore, given that $z \in H^{s+1}(\Omega)$ for some $s, 0 \leq s \leq p$, we have that

$$
\begin{aligned}
\mathrm{II}_{2} \leq & C_{i} \sum_{\kappa \in \mathcal{T}_{h}}\left\|h r_{h}\right\|_{L_{2}(\kappa)} \min _{0 \leq \sigma \leq s}\left\{h_{\kappa}^{\sigma}|z|_{H^{\sigma+1}(\hat{\kappa})}\right\} \\
\mathrm{III}_{2} \leq & C_{i} \sum_{\kappa \in \mathcal{T}_{h}}\left(\|\beta\|_{L_{\infty}(\kappa)}+h_{\kappa}\|\hat{c}\|_{L_{\infty}(\kappa)}\right)\left\|\delta r_{h}\right\|_{L_{2}(\kappa)} \min _{0 \leq \sigma \leq s}\left\{h_{\kappa}^{\sigma}|z|_{H^{\sigma+1}(\hat{\kappa})}\right\} \\
& +\sum_{\kappa \in \mathcal{T}_{h}}\left(\|\beta\|_{L_{\infty}(\kappa)}+h_{\kappa}\|\hat{c}\|_{L_{\infty}(\kappa)}\right)\left\|\delta r_{h}\right\|_{L_{2}(\kappa)}|z|_{H^{1}(\hat{\kappa})} .
\end{aligned}
$$

For the case of the stabilisation-dependent dual problem, $z$ is replaced by $z_{\delta}$ and estimates identical to those for $\mathrm{I}_{2}$ and $\mathrm{II}_{2}$ hold for $\mathrm{I}_{2, \delta}$ and $\mathrm{II}_{2, \delta}$, while in the corresponding bound for $\mathrm{III}_{2, \delta}$ the second sum on the right-hand side of the estimate for $\mathrm{III}_{2}$ does not arise.

We see from Lemma 14 and inequality (3.30) that when the analysis is based on the standard dual problem, the resulting bound on the error in the approximation of the functional $J(\cdot)$ is dominated by the term $\mathrm{III}_{2}$ which stems from the stabilisation in the numerical method.

As in Section 2, we further illustrate the features of our a posteriori error estimation by a simple example which concerns the finite element approximation of the normal flux through the outflow boundary $\Gamma_{+}$for the solution to the transport problem (3.1). Thus, assuming that $\psi \in L_{2}\left(\Gamma_{+}\right)$is a given weight function, we are interested in the quantity

$$
N_{\psi}(u)=\int_{\Gamma_{+}}(\beta \cdot \nu) u \psi \mathrm{ds}
$$


where $u$ is the solution to (3.1). Supposing that $u_{h}$ is the Galerkin approximation to $u$ defined by the stabilised finite element scheme (3.6), we wish to estimate the error between the computed outflow normal flux $N_{\psi}\left(u_{h}\right)$ and the actual value $N_{\psi}(u)$ by means of the two dual problems. In Section 4 we present a number of numerical experiments to compare the two approaches.

For this particular functional the dual problems (3.24), (3.25) in strong form read as follows: find $z$ such that

$$
\mathcal{L}^{*} z \equiv-\nabla \cdot(\beta z)+c z=0 \quad \text { in } \Omega, \quad z=\psi \quad \text { on } \Gamma_{+} ;
$$

for $\delta \in W_{\infty}^{1}(\Omega)$, find $z_{\delta} \in H(\Omega)$ such that

$$
\begin{array}{rll}
\mathcal{L}^{*}\left(z_{\delta}+\delta \hat{\mathcal{L}} z_{\delta}\right) & =0 & \text { in } \Omega, \\
\hat{\mathcal{L}} z_{\delta} & =0 & \text { on } \Gamma_{-}, \\
z_{\delta}+\delta \hat{\mathcal{L}} z_{\delta} & =\psi & \text { on } \Gamma_{+} .
\end{array}
$$

We shall now derive an a priori bound on the error in the computed functional in terms of Sobolev norms of the analytical solution $u$; this will indicate the expected rate of convergence for $\left|N_{\psi}(u)-N_{\psi}\left(u_{h}\right)\right|$ as $h$ tends to zero. We need the following result which is a minor variation on an a priori error bound from [10].

Lemma 15 Assume that (B) holds and let $u$ and $u_{h}$ denote the solutions of (3.1) and (3.6), respectively. Suppose that $u \in H^{s+1}(\Omega)$ for some $s, 0 \leq s \leq p$, and there exist positive constants $c_{\delta}$ and $C_{\delta}$ such that $c_{\delta} h \leq \delta \leq C_{\delta}$ on $\Omega$; then

$$
||\left|u-u_{h}\right||| \leq h^{s+1 / 2}|u|_{H^{s+1}(\Omega)},
$$

where

$$
\|\| v \mid\left\|^{2}=\right\| \sqrt{\delta} \mathcal{L} v\left\|_{L_{2}(\Omega)}^{2}+\right\| v\left\|_{L_{2}(\Omega)}^{2}+\right\| v\left\|_{L_{2}\left(\Gamma_{-}\right)}^{2}+\right\| v \|_{L_{2}\left(\Gamma_{+}\right)}^{2}
$$

and $C$ is a constant independent of the mesh function $h$.

Thereby, from Lemmas 14 and 15, we deduce the following a priori error bound.

Lemma 16 Assume that (B) holds and let $u$ and $u_{h}$ denote the solutions of (3.1) and (3.6), respectively. Given that $u \in H^{s+1}(\Omega)$ and $z \in H^{s+1}\left(\Gamma_{-}\right)$for some $s, 0 \leq s \leq p$, and there exist positive constants $c_{\delta}$ and $C_{\delta}$ such that $c_{\delta} h \leq \delta \leq C_{\delta} h$ on $\Omega$, we have that

$$
\mathrm{I}_{2} \leq C h^{2 s+3 / 2}|u|_{H^{s+1}(\Omega)}|z|_{H^{s+1}\left(\Gamma_{-}\right)} .
$$

Furthermore, given that $z \in H^{s+1}(\Omega)$ for some $s, 0 \leq s \leq p$, we have that

$$
\begin{aligned}
\mathrm{II}_{2} & \leq C h^{2 s+1}|u|_{H^{s+1}(\Omega)}|z|_{H^{s+1}(\Omega)}, \\
\mathrm{III}_{2} & \leq C h^{2 s+1}|u|_{H^{s+1}(\Omega)}|z|_{H^{s+1}(\Omega)}+C h^{2}|u|_{H^{s+1}(\Omega)}|z|_{H^{1}(\Omega)},
\end{aligned}
$$

where $C$ is a constant independent of the mesh function $h$. For the case of the stabilisationdependent dual problem, $z$ is replaced by $z_{\delta}$ and estimates identical to those for $\mathrm{I}_{2}$ and $\mathrm{II}_{2}$ hold for $\mathrm{I}_{2, \delta}$ and $\mathrm{II}_{2, \delta}$, while in the corresponding bound for $\mathrm{III}_{2, \delta}$ the second term on the right-hand side of the estimate for $\mathrm{III}_{2}$ does not arise. 
Thus, Lemma 16 indicates that (under suitable assumptions on the smoothness of $u$ and the dual solution $z$ ) the error bound in the outward normal flux based on the standard dual problem is of size $\mathcal{O}\left(h^{2}\right)$, irrespective of the degree $p$ of the polynomial approximation used in the finite element space $S_{h}$. On the other hand, using the error estimate based on the stabilisation-dependent dual problem we find that $\left|N_{\psi}(u)-N_{\psi}\left(u_{h}\right)\right|=\mathcal{O}\left(h^{2 s+1}\right)$ for $0 \leq s \leq p$, provided that $z_{\delta} \in H^{s+1}(\Omega)$ with $\left\|z_{\delta}\right\|_{H^{s+1}(\Omega)} \leq$ Const., independent of $h$.

Remark 17 We note that if we assume that $u \in H^{s+1}\left(\Gamma_{-}\right)$for some $s, 0 \leq s \leq p$, then the a priori error bound for the boundary term $\mathrm{I}_{2}$ stated in Lemma 16 may be improved provided that the boundary residual is redefined as $r_{h}^{-}=g-P_{h} g$. Indeed, on selecting $z_{h}=P_{h} z$ in (3.26a) with $P_{h}$ chosen as indicated at the end of Remark 7 and applying (B) we deduce that

$$
\mathrm{I}_{2} \leq C h^{2 s+2}|u|_{H^{s+1}\left(\Gamma_{-}\right)}|z|_{H^{s+1}\left(\Gamma_{-}\right)}
$$

provided that $z \in H^{s+1}\left(\Gamma_{-}\right)$. An analogous bound holds for $I_{2, \delta}$.

Remark 18 The absence of a forcing function in the first dual problem (3.34) means that the error representation formula (3.26a) may be re-written in the following form:

$$
\begin{aligned}
N_{\psi}(u)-N_{\psi}\left(u_{h}\right)= & -\left((\beta \cdot \nu) r_{h}^{-}, z-z_{h}\right)_{\Gamma_{-}}+\left(r_{h}, z-z_{h}\right)+\left(\delta r_{h}, \hat{\mathcal{L}}\left(z-z_{h}\right)\right) \\
& -\left(\delta r_{h},(\hat{c}+c-\nabla \cdot \beta) z\right) .
\end{aligned}
$$

Thus, if the negative-adjoint stabilisation is employed, i.e. $\hat{\mathcal{L}}:=-\mathcal{L}^{*}$, then

$$
\hat{c}+c-\nabla \cdot \beta=0 \quad \text { for all } x \text { in } \bar{\Omega},
$$

and it is easy to verify that the solutions to the two dual problems (3.34) and (3.35) coincide, i.e. $z \equiv z_{\delta}$. Consequently, when $\hat{\mathcal{L}}:=-\mathcal{L}^{*}$ the error representation formulas resulting from the two dual problems are identical; in particular, this is the case when the absorption term $c$ is identically zero and the velocity field $\beta$ is incompressible, i.e. $\nabla \cdot \beta=0$ for all $x$ in $\Omega$.

\section{Computational implementation; numerical experi- ments}

In this section we present a number of numerical experiments to compare the various error bounds which were based on the two dual problems, (3.34) and (3.35). In Examples 13 of Sections 4.3-4.5, we implemented (3.6) with the streamline diffusion stabilisation, corresponding to $\hat{c}=0$.

\subsection{Numerical approximation of $z_{\delta}$}

First, we outline the numerical method employed for the approximation of the analytical solution $z_{\delta}$ to the stabilisation-dependent dual problem (3.35); here, we exploit the stabilised finite element method introduced and analysed in [7]. Let $\mathcal{T}_{h_{d}}$ be an admissible 
subdivision of $\Omega$ into shape regular finite elements $\kappa$, with corresponding mesh function $h_{d}$. Further, we define $S_{h_{d}}$ as the finite element space consisting of continuous piecewise polynomials of fixed degree $p_{d}, p_{d} \geq 1$; for simplicity, in this paper we shall only consider the case where $p_{d}=p=1$.

Let us first rewrite the stabilisation-dependent dual problem (3.35) in the following strong form

$$
\mathcal{L}_{d} z_{\delta} \equiv-\nabla \cdot\left(a_{d} \nabla z_{\delta}\right)+b_{d} \cdot \nabla z_{\delta}+c_{d} z_{\delta}=0
$$

where

$$
a_{d}=\delta \beta \beta^{T}, \quad b_{d}=(\delta(c-\hat{c})-1) \beta, \quad c_{d}=\mathcal{L}^{*}(1+\delta \hat{c}) .
$$

We emphasise here that the matrix $\beta \beta^{T}$ is negative semi-definite, but not positive definite. At any rate, (4.1) is a second-order partial differential equation with nonnegative characteristic form. Now let us introduce the bilinear form $B_{\delta, \delta_{d}}(\cdot, \cdot)$ defined by

$$
\begin{aligned}
B_{\delta, \delta_{d}}(w, v)= & \left(a_{d} \nabla w, \nabla v\right)-\left(w, \nabla \cdot\left(b_{d} v\right)\right)+\left(c_{d} w, v\right) \\
& +\sum_{\kappa \in \mathcal{T}_{h_{d}}}\left(\hat{\mathcal{L}}_{d} w, \delta_{d} b_{d} \cdot \nabla v\right)_{\kappa}-((\beta \cdot \nu) w, v)_{\Gamma_{-}},
\end{aligned}
$$

and the linear functional

$$
l_{\delta_{d}}(v)=((\beta \cdot \nu) \psi, v)_{\Gamma_{+}},
$$

where, on element $\kappa \in \mathcal{T}_{h_{d}}$, we define $\hat{\mathcal{L}}_{d}$ by

$$
\hat{\mathcal{L}}_{d} w=-\nabla \cdot\left(P_{\kappa}\left(a_{d} \nabla w\right)\right)+b_{d} \cdot \nabla w+c_{d} w,
$$

and $P_{\kappa}$ signifies the orthogonal projection in $\left[L_{2}(\kappa)\right]^{n}$ onto $\left[\mathcal{P}_{p_{d}-1}(\kappa)\right]^{n}$. Here, the dual stabilisation parameter $\delta_{d}$ is chosen as the positive piecewise constant function

$$
\delta_{d}=C_{d} h_{d}
$$

where $\left.h_{d}\right|_{\kappa}=\operatorname{diam}(\kappa)$ for $\kappa \in \mathcal{T}_{h_{d}}$. The finite element approximation to $z_{\delta}$ is then defined as follows (cf. [7]): find $\hat{z}_{\delta} \in S_{h_{d}}$ such that

$$
B_{\delta, \delta_{d}}\left(\hat{z}_{\delta}, v_{h_{d}}\right)=l_{\delta_{d}}\left(v_{h_{d}}\right) \quad \forall v_{h_{d}} \in S_{h_{d}}
$$

The next section is devoted to the description of the adaptive finite element algorithm.

\subsection{Adaptive Algorithm}

Following from Theorem 12, we have the a posteriori error bounds:

$$
\left|N_{\psi}(u)-N_{\psi}\left(u_{h}\right)\right| \leq \begin{cases}\epsilon_{2}\left(u_{h}, z, z_{h}\right) & \text { for dual problem }(3.34) \\ \epsilon_{2, \delta}\left(u_{h}, z_{\delta}, z_{h, \delta}\right) & \text { for dual problem }(3.35)\end{cases}
$$


where, as in (3.31),

$$
\begin{aligned}
\epsilon_{2} & =\sum_{\kappa \in \mathcal{T}_{h}}\left|-\left((\beta \cdot \nu) r_{h}^{-},\left(z-z_{h}\right)\right)_{\partial \kappa \cap \Gamma_{-}}+\left(r_{h}, z-z_{h}\right)_{\kappa}-\left(r_{h}, \delta \hat{\mathcal{L}} z_{h}\right)_{\kappa}\right| \\
\epsilon_{2, \delta} & =\sum_{\kappa \in \mathcal{T}_{h}}\left|-\left((\beta \cdot \nu) r_{h}^{-},\left(z_{\delta}-z_{\delta, h}\right)\right)_{\partial \kappa \cap \Gamma_{-}}+\left(r_{h}, z_{\delta}-z_{\delta, h}\right)_{\kappa}+\left(r_{h}, \delta \hat{\mathcal{L}}\left(z_{\delta}-z_{\delta, h}\right)\right)_{\kappa}\right| .
\end{aligned}
$$

With this notation, we now consider the problem of designing a mesh such that

$$
\epsilon_{2, \delta}\left(u_{h}, z_{\delta}, z_{h, \delta}\right) \leq T O L
$$

subject to the constraint that the number $N$ of elements is minimised. To this end, we adopt the optimised mesh strategy outlined in [13]. Defining

$$
\begin{aligned}
& A(x)=\left|r_{h}\left(z_{\delta}-z_{\delta, h}\right)+\delta r_{h} \hat{\mathcal{L}}\left(z_{\delta}-z_{h, \delta}\right)\right| / h_{\Omega}^{3}(x), \\
& B(x)=\left|(\beta \cdot \nu)\left(g-u_{h}\right)\left(z_{\delta}-z_{\delta, h}\right)\right| / h_{\Gamma_{-}}^{4}(x),
\end{aligned}
$$

where $h_{\Omega}$ and $h_{\Gamma_{-}}$are the mesh functions on $\Omega \cap \Gamma_{+}$and $\Gamma_{-}$, respectively, after an elementary calculation involving a Lagrange multiplier $\lambda$, we arrive at the optimal mesh size distributions $h_{\Omega}^{\mathrm{opt}}(x)$ and $h_{\Gamma_{-}}^{\mathrm{opt}}(x)$ given by

$$
h_{\Omega}^{\mathrm{opt}}=\left(\frac{2}{3 \lambda A}\right)^{1 / 5}, \quad h_{\Gamma_{-}}^{\mathrm{opt}}=\left(\frac{1}{4 \lambda B}\right)^{1 / 5},
$$

where $\lambda$ is the positive root of

$$
\left(\frac{2}{3 \lambda}\right)^{3 / 5} \int_{\Omega} A^{2 / 5} \mathrm{~d} x+\left(\frac{1}{4 \lambda}\right)^{4 / 5} \int_{\Gamma_{-}} B^{1 / 5} \mathrm{~d} s=T O L
$$

For $T O L \ll 1$, we anticipate $\lambda \gg 1$, so that $(1 / \lambda)^{4 / 5} \ll(1 / \lambda)^{3 / 5}$. Thus, for simplicity, we neglect the boundary integral term in (4.4); on eliminating $\lambda$, we then arrive at the following explicit formula for $h_{\Omega}^{\mathrm{opt}}(x)$ :

$$
h_{\Omega}^{\mathrm{opt}}(x) \approx\left(\frac{T O L}{W}\right)^{1 / 3} \frac{1}{A^{1 / 5}(x)}, \quad \text { where } \quad W=\int_{\Omega} A^{2 / 5}(x) \mathrm{d} x .
$$

In order to construct a computational mesh with granularity predicted by (4.5), we employ the red-green isotropic refinement strategy. Here, the user must first specify a (coarse) background mesh upon which any future refinement will be based. A red refinement corresponds to dividing a certain triangle into four similar triangles by connecting the midpoints of the sides. Green refinement is only temporary and is used to remove any hanging nodes caused by a red refinement. We note that green refinement is only applied on elements which have one hanging node; for elements with two or more hanging nodes a red refinement is performed. Within this mesh modification strategy, elements may also be removed from the mesh (i.e. derefined) provided they do not lie in the original background mesh.

For the practical implementation of this adaptive algorithm, the analytical solution $z_{\delta}$ to the stabilisation-dependent dual problem (3.35) will be approximated as outlined in the 


\begin{tabular}{||c|c|c|c|c||}
\hline Mesh & $\left\|u-u_{h}\right\|_{L_{2}(\Omega)}$ & $\mathrm{k}$ & $\left|N_{\psi}(u)-N_{\psi}\left(u_{h}\right)\right|$ & $\mathrm{k}$ \\
\hline $17 \times 17$ & $2.077 \times 10^{-3}$ & - & $3.419 \times 10^{-5}$ & - \\
\hline $33 \times 33$ & $4.343 \times 10^{-4}$ & 2.26 & $4.461 \times 10^{-6}$ & 2.94 \\
\hline $65 \times 65$ & $1.021 \times 10^{-4}$ & 2.09 & $5.657 \times 10^{-7}$ & 2.98 \\
\hline $129 \times 129$ & $2.500 \times 10^{-5}$ & 2.03 & $7.107 \times 10^{-8}$ & 2.99 \\
\hline $257 \times 257$ & $6.208 \times 10^{-6}$ & 2.01 & $8.901 \times 10^{-9}$ & 3.00 \\
\hline
\end{tabular}

Table 1: Example 1. Convergence of $\left\|u-u_{h}\right\|_{L_{2}(\Omega)}$ and $\left|N_{\psi}(u)-N_{\psi}\left(u_{h}\right)\right|$ with $\delta=h / 4$.

\begin{tabular}{||c|c|c|c|c|c|c||}
\hline Mesh & $\mathrm{I}_{1}$ & $\mathrm{k}$ & $\mathrm{II}_{1}$ & $\mathrm{k}$ & $\mathrm{III}_{1}$ & $\mathrm{k}$ \\
\hline $17 \times 17$ & $1.804 \times 10^{-6}$ & - & $2.511 \times 10^{-6}$ & - & $3.490 \times 10^{-5}$ & - \\
\hline $33 \times 33$ & $1.119 \times 10^{-7}$ & 4.01 & $1.568 \times 10^{-7}$ & 4.00 & $4.506 \times 10^{-6}$ & 2.95 \\
\hline $65 \times 65$ & $7.147 \times 10^{-9}$ & 3.97 & $1.003 \times 10^{-8}$ & 3.97 & $5.686 \times 10^{-7}$ & 2.99 \\
\hline $129 \times 129$ & $4.552 \times 10^{-10}$ & 3.97 & $6.379 \times 10^{-10}$ & 3.97 & $7.126 \times 10^{-8}$ & 2.97 \\
\hline $257 \times 257$ & $2.876 \times 10^{-11}$ & 3.98 & $4.026 \times 10^{-11}$ & 3.99 & $8.913 \times 10^{-9}$ & 3.00 \\
\hline
\end{tabular}

Table 2: Example 1. Convergence of the terms in the error representation formula with $\delta=h / 4$.

previous section. For clarity, we define $\hat{\epsilon}_{2, \delta}=\epsilon_{2, \delta}\left(u_{h}, \hat{z}_{\delta}, \hat{z}_{h, \delta}\right)$, where $\hat{z}_{h, \delta}$ is the interpolant of $\hat{z}_{\delta}$ onto the mesh $\mathcal{T}_{h}$ used to calculate the approximation $u_{h}$ to the primal problem (3.1). The mesh $\mathcal{T}_{h_{d}}$ for the dual problem (3.35) will be constructed via the fixed fraction strategy outlined in [13] using the local error indicator $\left\|h_{d} \hat{r}_{h_{d}}\right\|_{L_{2}(\kappa)}$ as in [6], where $\hat{r}_{h_{d}}$ is defined to be the residual of the computed dual solution $\hat{z}_{\delta}$.

\subsection{Example 1}

In this example we first investigate the order of convergence of the error in the outflow normal flux as the mesh is uniformly refined. To simplify the presentation, we consider a model problem which ensures that the solutions to the two dual problems, (3.34) and (3.35), are identical, cf. Remark 18. To this end, we let $\beta=(1+\sin (\pi y), 2), c=0$ and $f=0$ with boundary condition

$$
u(x, y)= \begin{cases}1-y^{5} & \text { for } x=0,0 \leq y \leq 1 \\ \mathrm{e}^{-50 x^{4}} & \text { for } 0 \leq x \leq 1, y=0 .\end{cases}
$$

In addition, we define the weight function $\psi$ in the functional $N_{\psi}(\cdot)$, cf. $(3.33)$, by

$$
\psi= \begin{cases}1-\sin ^{2}(\pi(1-y) / 2) \cos (\pi y / 2) & \text { for } x=1,0 \leq y \leq 1 \\ 1-(1-x)^{3}-(1-x)^{4} / 2 & \text { for } 0 \leq x \leq 1, y=1\end{cases}
$$

Thus, the true value of the outflow normal flux is $N_{\psi}(u)=1.8664$. 


\begin{tabular}{||c|c|c|c|c||}
\hline Mesh & $\left\|u-u_{h}\right\|_{L_{2}(\Omega)}$ & $\mathrm{k}$ & $\left|N_{\psi}(u)-N_{\psi}\left(u_{h}\right)\right|$ & $\mathrm{k}$ \\
\hline $17 \times 17$ & $8.442 \times 10^{-3}$ & - & $2.831 \times 10^{-4}$ & - \\
\hline $33 \times 33$ & $7.876 \times 10^{-4}$ & 3.42 & $1.963 \times 10^{-5}$ & 3.85 \\
\hline $65 \times 65$ & $1.099 \times 10^{-4}$ & 2.84 & $1.252 \times 10^{-6}$ & 3.97 \\
\hline $129 \times 129$ & $2.504 \times 10^{-5}$ & 2.13 & $7.854 \times 10^{-8}$ & 3.99 \\
\hline $257 \times 257$ & $6.197 \times 10^{-6}$ & 2.01 & $4.912 \times 10^{-9}$ & 4.00 \\
\hline
\end{tabular}

Table 3: Example 1. Convergence of the terms in the error representation formula with $\delta=25 h^{2}$.

\begin{tabular}{||c|c|c|c|c|c|c||}
\hline Mesh & $\mathrm{I}_{1}$ & $\mathrm{k}$ & $\mathrm{II}_{1}$ & $\mathrm{k}$ & $\mathrm{III}_{1}$ & $\mathrm{k}$ \\
\hline $17 \times 17$ & $2.671 \times 10^{-6}$ & - & $3.276 \times 10^{-6}$ & - & $2.837 \times 10^{-4}$ & - \\
\hline $33 \times 33$ & $1.358 \times 10^{-7}$ & 4.30 & $1.657 \times 10^{-7}$ & 4.31 & $1.966 \times 10^{-5}$ & 3.85 \\
\hline $65 \times 65$ & $7.210 \times 10^{-9}$ & 4.24 & $9.945 \times 10^{-9}$ & 4.06 & $1.255 \times 10^{-6}$ & 3.97 \\
\hline $129 \times 129$ & $4.549 \times 10^{-10}$ & 3.99 & $6.373 \times 10^{-10}$ & 3.96 & $7.873 \times 10^{-8}$ & 3.99 \\
\hline $257 \times 257$ & $2.883 \times 10^{-11}$ & 3.98 & $4.037 \times 10^{-11}$ & 3.98 & $4.924 \times 10^{-9}$ & 4.00 \\
\hline
\end{tabular}

Table 4: Example 1. Convergence of the terms in the error representation formula with $\delta=25 h^{2}$.

In Table 1 we show $\left\|u-u_{h}\right\|_{L_{2}(\Omega)}$ and $\left|N_{\psi}(u)-N_{\psi}\left(u_{h}\right)\right|$, along with their respective rates of convergence $k$, on a sequence of uniform triangular meshes: in each case the mesh is constructed from a uniform $N \times N$ mesh by connecting the bottom-left corner of each mesh square with its top-right corner. Here, we observe that with the stabilisation parameter chosen to be $h / 4$, the $L_{2}(\Omega)$ norm of the error converges like $\mathcal{O}\left(h^{2}\right)$ and the error in the functional, $N_{\psi}\left(u-u_{h}\right)$, converges like $\mathcal{O}\left(h^{3}\right)$ as expected, cf. Lemma 16 with $s=p=1$. However, if we now look at the convergence rates of each of the terms in the error representation formula (3.26a) (or (3.26b)), cf. Definition 13, then we observe, cf. Table 2, that the first two terms, i.e. terms $\mathrm{I}_{1}\left(\equiv \mathrm{I}_{1, \delta}\right)$ and $\mathrm{II}_{1}\left(\equiv \mathrm{II}_{1, \delta}\right)$, both converge like $\mathcal{O}\left(h^{4}\right)$ as $h$ tends to zero. We note that term $\mathrm{II}_{1}$ is super-convergent, since we only expect to observe a convergence rate of $\mathcal{O}\left(h^{3}\right)$ as $h$ tends to zero, cf. Lemma 16. Thereby, the whole bound is dominated by the third term, which arises as a result of the stabilisation employed. Furthermore, we note that the same asymptotic rates of convergence are achieved for each of the above quantities on unstructured quasi-uniform triangular meshes as well; this indicates that the super-convergence of term $\mathrm{II}_{1}$ is robust with respect to mesh distortion.

Thus while classical a priori error analysis, cf. [10], indicates that the stabilisation should be chosen so that $\delta=\mathcal{O}(h)$ as the mesh is refined, in order to obtain optimal error estimates in a mesh dependent norm, cf. Lemma 15; here, our numerical experiments indicate that $\delta$ should be chosen to be $\delta=\mathcal{O}\left(h^{2}\right)$ in order to obtain $\mathcal{O}\left(h^{4}\right)$ convergence for the error in outward normal flux. Indeed, in Tables 3 and 4 we repeat the above numerical experiments with $\delta=25 h^{2}$. From Table 3, we observe that the $L_{2}(\Omega)$ norm of the error still approaches second order as the mesh is refined, while the error in the functional $N_{\psi}(\cdot)$ 


\begin{tabular}{||c|c|c|c|c||}
\hline Mesh & $\left\|u-u_{h}\right\|_{L_{2}(\Omega)}$ & $\mathrm{k}$ & $\left|N_{\psi}(u)-N_{\psi}\left(u_{h}\right)\right|$ & $\mathrm{k}$ \\
\hline $17 \times 17$ & $2.927 \times 10^{-3}$ & - & $2.957 \times 10^{-4}$ & - \\
\hline $33 \times 33$ & $5.195 \times 10^{-4}$ & 2.49 & $3.860 \times 10^{-5}$ & 2.94 \\
\hline $65 \times 65$ & $1.079 \times 10^{-4}$ & 2.27 & $4.944 \times 10^{-6}$ & 2.96 \\
\hline $129 \times 129$ & $2.544 \times 10^{-5}$ & 2.08 & $6.257 \times 10^{-7}$ & 2.98 \\
\hline $257 \times 257$ & $6.260 \times 10^{-6}$ & 2.02 & $7.874 \times 10^{-8}$ & 2.99 \\
\hline
\end{tabular}

Table 5: Example 2. Convergence of $\left\|u-u_{h}\right\|_{L_{2}(\Omega)}$ and $\left|N_{\psi}(u)-N_{\psi}\left(u_{h}\right)\right|$ with $\delta=h / 4$.

is now clearly $\mathcal{O}\left(h^{4}\right)$ as $h$ tends to zero. Furthermore, from Table 4 we now clearly observe that all three terms in the error representation formula (3.26a) (or (3.26b)) are fourth order convergent as the mesh is refined. As before, the same asymptotic behaviour is achieved on unstructured triangular meshes also.

In summary, this numerical experiment indicates that when the quantity of interest is the outward normal flux of the solution, the stabilisation parameter $\delta$ should not be chosen to be $\mathcal{O}(h)$ as advocated on grounds of accuracy by standard a priori error analysis in the mesh-dependent norm $\|\cdot \cdot\|$. Indeed, due to the inherent super-convergence property of term $\mathrm{II}_{1}$ (and $\mathrm{II}_{1, \delta}$ ), cf. Definition 13, the error in the outflow flux can be reduced to $\mathcal{O}\left(h^{4}\right)$, for a piecewise linear approximation $u_{h}$ to $u$, provided that $\delta=C_{\delta} h^{2}$. However, a drawback of such a stabilisation is that the resulting system of linear equations becomes increasingly difficult to solve as $h$ is reduced, unless $C_{\delta}$ is chosen sufficiently large; this, however, is rather counter-productive, since a large $C_{\delta}$ leads to much larger error in the computed functional. Thus, while $\left|N_{\psi}(u)-N_{\psi}\left(u_{h}\right)\right|$ is now $\mathcal{O}\left(h^{4}\right)$, the constant that multiplies the powers of $h$ is increased; thereby on practical meshes, we would still advocate using $\mathcal{O}(h)$ stabilisation with a 'small' $C_{\delta}$, at the expense of suboptimal convergence rates in the functional of interest.

\subsection{Example 2}

In this example we consider a compressible problem in order to highlight the main differences between using the standard dual problem (3.34) and the stabilisation-dependent dual problem (3.35). To this end, we let $\beta=(1+x, 1+y), c=0$ and $f=0$ with same boundary conditions as employed in Example 1; i.e.

$$
u(x, y)= \begin{cases}1-y^{5} & \text { for } x=0,0 \leq y \leq 1 \\ \mathrm{e}^{-50 x^{4}} & \text { for } 0 \leq x \leq 1, y=0\end{cases}
$$

Selecting the weight function $\psi$ as in equation (4.6), we have $N_{\psi}(u)=2.4676$.

In Table 5 we first investigate the order of convergence of the error in the functional $N_{\psi}(\cdot)$ as $h$ tends to zero. As in Example 1, we observe that $\left|N_{\psi}(u)-N_{\psi}\left(u_{h}\right)\right|$ converges like $\mathcal{O}\left(h^{3}\right)$ with $\mathcal{O}(h)$ stabilisation, while the $L_{2}(\Omega)$ norm of the error is of second order. In Tables 6 and 7 we show the convergence of each of the terms in the error representation formulas (3.26a) and (3.26b), respectively. Table 6 again shows that the second term in the 


\begin{tabular}{||c|c|c|c|c|c|c||}
\hline Mesh & $\mathrm{I}_{1}$ & $\mathrm{k}$ & $\mathrm{II}_{1}$ & $\mathrm{k}$ & $\mathrm{III}_{1}$ & $\mathrm{k}$ \\
\hline $17 \times 17$ & $3.308 \times 10^{-6}$ & - & $3.350 \times 10^{-6}$ & - & $2.957 \times 10^{-4}$ & - \\
\hline $33 \times 33$ & $1.906 \times 10^{-7}$ & 4.12 & $2.295 \times 10^{-7}$ & 3.87 & $3.864 \times 10^{-5}$ & 2.94 \\
\hline $65 \times 65$ & $1.166 \times 10^{-8}$ & 4.03 & $1.515 \times 10^{-8}$ & 3.92 & $4.947 \times 10^{-6}$ & 2.97 \\
\hline $129 \times 129$ & $7.236 \times 10^{-10}$ & 4.01 & $9.742 \times 10^{-10}$ & 3.96 & $6.260 \times 10^{-7}$ & 2.98 \\
\hline $257 \times 257$ & $4.511 \times 10^{-11}$ & 4.00 & $6.178 \times 10^{-11}$ & 3.99 & $7.872 \times 10^{-8}$ & 2.99 \\
\hline
\end{tabular}

Table 6: Example 2. Convergence of the terms in the error representation formula (3.26a) with $\delta=h / 4$.

\begin{tabular}{||c|c|c|c|c|c|c||}
\hline Mesh & $\mathrm{I}_{1, \delta}$ & $\mathrm{k}$ & $\mathrm{II}_{1, \delta}$ & $\mathrm{k}$ & $\mathrm{III}_{1, \delta}$ & $\mathrm{k}$ \\
\hline $17 \times 17$ & $3.308 \times 10^{-6}$ & - & $1.501 \times 10^{-5}$ & - & $2.773 \times 10^{-4}$ & - \\
\hline $33 \times 33$ & $1.906 \times 10^{-7}$ & 4.12 & $2.041 \times 10^{-6}$ & 2.88 & $3.637 \times 10^{-5}$ & 2.93 \\
\hline $65 \times 65$ & $1.166 \times 10^{-8}$ & 4.03 & $2.562 \times 10^{-7}$ & 2.99 & $4.676 \times 10^{-6}$ & 2.96 \\
\hline $129 \times 129$ & $7.236 \times 10^{-10}$ & 4.01 & $3.166 \times 10^{-8}$ & 3.02 & $5.933 \times 10^{-7}$ & 2.98 \\
\hline $257 \times 257$ & $4.511 \times 10^{-11}$ & 4.00 & $3.919 \times 10^{-9}$ & 3.01 & $7.474 \times 10^{-8}$ & 2.99 \\
\hline
\end{tabular}

Table 7: Example 2. Convergence of the terms in the error representation formula (3.26b) with $\delta=h / 4$.

error representation formula (3.26a), i.e. term $\mathrm{II}_{1}$, is super-convergent; here, $\mathrm{II}_{1}=\mathcal{O}\left(h^{4}\right)$ as $h$ tends to zero. Furthermore, since the two dual problems are no longer equivalent, the third term in the error representation formula (3.26a) is only expected to converge like $\mathcal{O}\left(h^{2}\right)$ as $h$ tends to zero, cf. Lemma 16. Table 6 indicates that term III $_{1}$ is also superconvergent. However, as in Example 1, the error in the outward normal flux is entirely dominated by this term which arises as a result of the stabilisation employed. On the other hand, from Table 7 , we see that while the first term, term $\mathrm{I}_{1, \delta}$, in the error representation formula based on the stabilisation-dependent dual problem (cf. (3.26b)) is still fourth order convergent as the mesh is refined, the second term, term $\mathrm{II}_{1, \delta}$, is now only $\mathcal{O}\left(h^{3}\right)$ as $h$ tends to zero. Thus, by employing the stabilisation-dependent dual problem (3.35) the terms in the error representation formula involving integrations over the entire computational domain $\Omega$, i.e. terms $\mathrm{II}_{1, \delta}$ and $\mathrm{III}_{1, \delta}$, are 'balanced'. This will be essential for ensuring that any a posteriori error estimate derived by further bounding the terms on the righthand side of (3.29) from above, converges at the same asymptotic rate as the true error in the functional $N_{\psi}(\cdot)$. Indeed, in Table 8 we now compare the convergence rates of the a posteriori error bounds $\epsilon_{2}$ and $\epsilon_{2, \delta}$, cf. (4.3) and (3.30), together with their respective effectivity indices $\theta_{1}$ and $\theta_{2}$; here $\theta_{1}=\epsilon_{2} /\left|N_{\psi}(u)-N_{\psi}\left(u_{h}\right)\right|$ and $\theta_{2}=\epsilon_{2, \delta} /\left|N_{\psi}(u)-N_{\psi}\left(u_{h}\right)\right|$. Here, we see that the error bound $\epsilon_{2}$ now only converges at the sub-optimal rate of $\mathcal{O}\left(h^{2}\right)$ as $h$ tends to zero; this leads to large effectivity indices which grow as the mesh is refined. Table 9 shows each of the terms in the error bound $\epsilon_{2}$ together with their respective rates of convergence, cf. Definition 13. Here, we see that while term $\mathrm{I}_{2}$ still converges like $\mathcal{O}\left(h^{4}\right)$ 


\begin{tabular}{||c|c|c|c|c|c|c||}
\hline Mesh & $\epsilon_{2}$ & $k$ & $\theta_{1}$ & $\epsilon_{2, \delta}$ & $k$ & $\theta_{2}$ \\
\hline $17 \times 17$ & $3.195 \times 10^{-3}$ & - & 10.8 & $3.724 \times 10^{-4}$ & - & 1.26 \\
\hline $33 \times 33$ & $8.150 \times 10^{-4}$ & 1.97 & 21.1 & $5.058 \times 10^{-5}$ & 2.88 & 1.31 \\
\hline $65 \times 65$ & $2.043 \times 10^{-4}$ & 2.00 & 41.3 & $6.678 \times 10^{-6}$ & 2.92 & 1.35 \\
\hline $129 \times 129$ & $5.108 \times 10^{-5}$ & 2.00 & 81.6 & $8.622 \times 10^{-7}$ & 2.95 & 1.38 \\
\hline $257 \times 257$ & $1.277 \times 10^{-5}$ & 2.00 & 162.2 & $1.279 \times 10^{-7}$ & 2.75 & 1.62 \\
\hline
\end{tabular}

Table 8: Example 2. Comparison of the a posteriori error bounds $\epsilon_{2}$ and $\epsilon_{2, \delta}$ with $\delta=h / 4$.

\begin{tabular}{||c|c|c|c|c|c|c||}
\hline Mesh & $\mathrm{I}_{2}$ & $\mathrm{k}$ & $\mathrm{II}_{2}$ & $\mathrm{k}$ & $\mathrm{III}_{2}$ & $\mathrm{k}$ \\
\hline $17 \times 17$ & $1.006 \times 10^{-5}$ & - & $7.431 \times 10^{-5}$ & - & $3.203 \times 10^{-3}$ & - \\
\hline $33 \times 33$ & $4.944 \times 10^{-7}$ & 4.35 & $9.117 \times 10^{-6}$ & 3.03 & $8.159 \times 10^{-4}$ & 1.97 \\
\hline $65 \times 65$ & $2.850 \times 10^{-8}$ & 4.12 & $1.138 \times 10^{-6}$ & 3.00 & $2.044 \times 10^{-4}$ & 2.00 \\
\hline $129 \times 129$ & $1.734 \times 10^{-9}$ & 4.04 & $1.422 \times 10^{-7}$ & 3.00 & $5.110 \times 10^{-5}$ & 2.00 \\
\hline $257 \times 257$ & $1.075 \times 10^{-10}$ & 4.01 & $1.777 \times 10^{-8}$ & 3.00 & $1.278 \times 10^{-5}$ & 2.00 \\
\hline
\end{tabular}

Table 9: Example 2. Convergence of the terms in the a posteriori error bound $\epsilon_{2}$ with $\delta=h / 4$.

as $h$ tends to zero, and term $\mathrm{II}_{2}$ is third-order convergent, $\mathrm{III}_{2}$ is now only second order convergent, in agreement with Lemma 16.

In contrast, by using the stabilisation-dependent dual problem, the error bound $\epsilon_{2, \delta}$ converges to zero at the same asymptotic rate as the true error in the functional $N_{\psi}(\cdot)$, thereby giving rise to small effectivity indices of approximate size 1.3-1.6 on all of the meshes considered, cf. Table 8. Moreover, from Table 10 we see that all the terms in the error bound $\epsilon_{2, \delta}$ converge to zero at the same rate as those in the error representation formula (3.26b); i.e. term $\mathrm{I}_{2, \delta}$ is fourth order convergent and both term $\mathrm{II}_{2, \delta}$ and term $\mathrm{III}_{2, \delta}$ converge like $\mathcal{O}\left(h^{3}\right)$ as $h$ tends to zero. Finally, we note that as in Example 1, the same asymptotic behaviour is achieved on unstructured quasi-uniform triangular meshes.

\begin{tabular}{||c|c|c|c|c|c|c||}
\hline Mesh & $\mathrm{I}_{2, \delta}$ & $\mathrm{k}$ & $\mathrm{II}_{2, \delta}$ & $\mathrm{k}$ & $\mathrm{III}_{2, \delta}$ & $\mathrm{k}$ \\
\hline $17 \times 17$ & $1.006 \times 10^{-5}$ & - & $1.943 \times 10^{-4}$ & - & $3.427 \times 10^{-4}$ & - \\
\hline $33 \times 33$ & $4.944 \times 10^{-7}$ & 4.35 & $2.870 \times 10^{-5}$ & 2.76 & $4.639 \times 10^{-5}$ & 2.89 \\
\hline $65 \times 65$ & $2.850 \times 10^{-8}$ & 4.12 & $3.912 \times 10^{-6}$ & 2.88 & $6.003 \times 10^{-6}$ & 2.95 \\
\hline $129 \times 129$ & $1.734 \times 10^{-9}$ & 4.04 & $5.125 \times 10^{-7}$ & 2.93 & $7.634 \times 10^{-7}$ & 2.98 \\
\hline $257 \times 257$ & $1.075 \times 10^{-10}$ & 4.01 & $6.578 \times 10^{-8}$ & 2.96 & $1.138 \times 10^{-7}$ & 2.75 \\
\hline
\end{tabular}

Table 10: Example 2. Convergence of the terms in the a posteriori error bound $\epsilon_{2, \delta}$ with $\delta=h / 4$. 


\begin{tabular}{||c|c|c|c|c|c|c||}
\hline \multicolumn{5}{|c|}{ Uniform Meshes } & \multicolumn{2}{c||}{ Unstructured Meshes } \\
\hline Mesh & $\left\|u-u_{h}\right\|_{L_{2}(\Omega)}$ & $\mathrm{k}$ & $\left|N_{\psi}\left(u-u_{h}\right)\right|$ & $\mathrm{k}$ & $\left|N_{\psi}\left(u-u_{h}\right)\right|$ & $\mathrm{k}$ \\
\hline $17 \times 17$ & $1.176 \times 10^{-1}$ & - & $3.234 \times 10^{-4}$ & - & $4.814 \times 10^{-4}$ & - \\
\hline $33 \times 33$ & $8.951 \times 10^{-2}$ & 0.39 & $2.191 \times 10^{-5}$ & 3.88 & $1.069 \times 10^{-4}$ & 2.17 \\
\hline $65 \times 65$ & $6.858 \times 10^{-2}$ & 0.38 & $1.264 \times 10^{-6}$ & 4.12 & $1.527 \times 10^{-5}$ & 2.81 \\
\hline $129 \times 129$ & $5.280 \times 10^{-2}$ & 0.38 & $3.509 \times 10^{-8}$ & 5.17 & $1.847 \times 10^{-6}$ & 3.05 \\
\hline $257 \times 257$ & $4.010 \times 10^{-2}$ & 0.40 & $5.638 \times 10^{-9}$ & 2.64 & $1.941 \times 10^{-7}$ & 3.25 \\
\hline
\end{tabular}

Table 11: Example 3: Convergence of $\left\|u-u_{h}\right\|_{L_{2}(\Omega)}$ and $\left|N_{\psi}(u)-N_{\psi}\left(u_{h}\right)\right|$ with $\delta=h / 4$.

\subsection{Example 3}

In this final example we consider a strongly compressible linear hyperbolic problem with discontinuous inflow boundary condition. To this end, we let $\beta=\left(10 y^{2}-12 x+1,1+y\right)$, $c=0$ and $f=0$. Here, the characteristics enter the computational domain $\Omega$ from three sides of $\partial \Omega$, namely from $x=0, y=0$ and $x=1$, and exit $\Omega$ through $y=1$. Thereby, we may prescribe the following boundary condition

$$
u(x, y)= \begin{cases}0 & \text { for } x=0,0.5<y \leq 1 \\ 1 & \text { for } x=0,0<y \leq 0.5 \\ 1 & \text { for } 0 \leq x \leq 0.5, y=0 \\ 0 & \text { for } 0.5<x \leq 1, y=0 \\ \sin ^{2}(\pi y) & \text { for } x=1,0 \leq y \leq 1\end{cases}
$$

the analytical solution to this problem is shown in Figure 1(a). We define the weight function $\psi$ in the functional $N_{\psi}(\cdot)$, cf. (3.33), by

$$
\psi=\sin (\pi x / 2) \text { for } 0 \leq x \leq 1, y=1 \text {. }
$$

Thus, the true value of the outward normal flux is $N_{\psi}(u)=0.24650$.

In Table 11 we investigate the asymptotic behaviour of $\left\|u-u_{h}\right\|_{L_{2}(\Omega)}$ and $\mid N_{\psi}(u)-$ $N_{\psi}\left(u_{h}\right) \mid$ as the mesh function $h$ tends to zero. Here, we see that on uniform triangular meshes, the convergence rate of the $L_{2}(\Omega)$ norm of the error is $k \approx 0.4$ due to the presence of the two discontinuities in the solution, cf. [6]; the convergence rate of the $L_{2}(\Omega)$ norm of the error on unstructured meshes is identical, so these results have been omitted. In contrast, the error in the outward normal flux is $\mathcal{O}\left(h^{3}\right)$ on the unstructured meshes and $\mathcal{O}\left(h^{4}\right)$ on uniform meshes. We note that there is a sudden increase in the order of convergence in the error in the functional on the uniform meshes followed by a decrease. This behaviour is caused by a change of sign in the boundary term in the error representation formula (3.26a); indeed, by calculating the convergence rate $k$ between the error in the outward normal flux on the $65 \times 65$ mesh and the $257 \times 257$ mesh gives rise to a convergence rate of $k=3.90$.

We remark that the high-order convergence attained for the error in the outward normal flux is attributed to the fact that while the residual terms $r_{h}$ and $r_{h}^{-}$are clearly large in the 
(a)
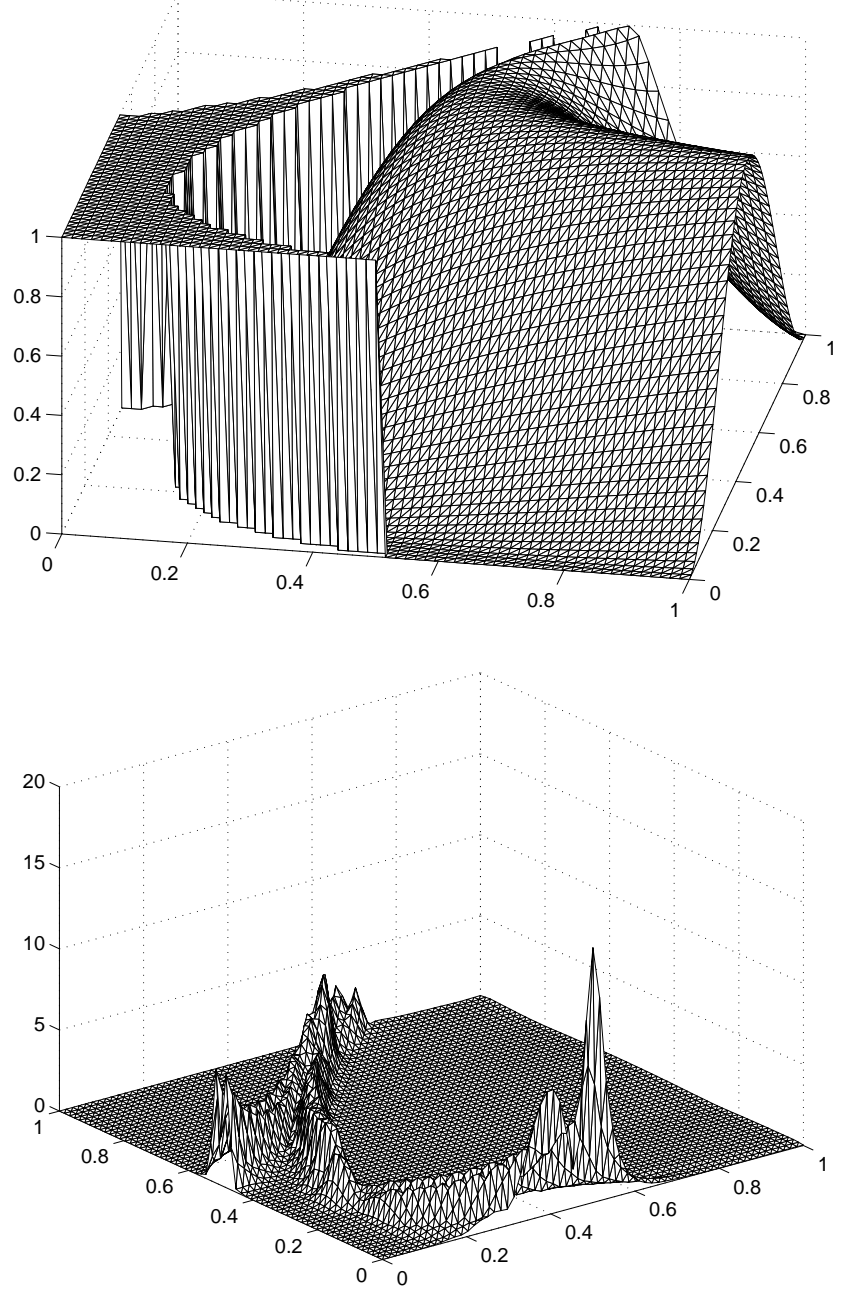

(b)

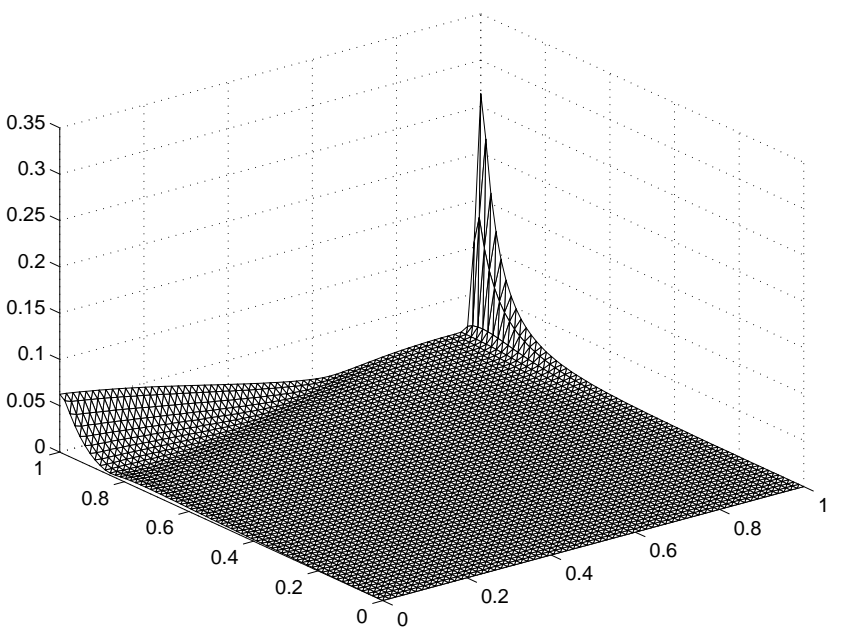

Figure 1: Example 3. (a) Analytical solution to the primal problem; (b) Scaled residual term $\left\|r_{h}\right\|_{L_{2}(\kappa)} / h_{\kappa}$ on a $65 \times 65$ mesh with $\delta=h / 4 ;$ (c) Scaled weighting term $\| z_{\delta}-$ $z_{\delta, h} \|_{L_{2}(\kappa)} / h_{\kappa}^{2}$ on a $65 \times 65$ mesh with $\delta=h / 4$. 


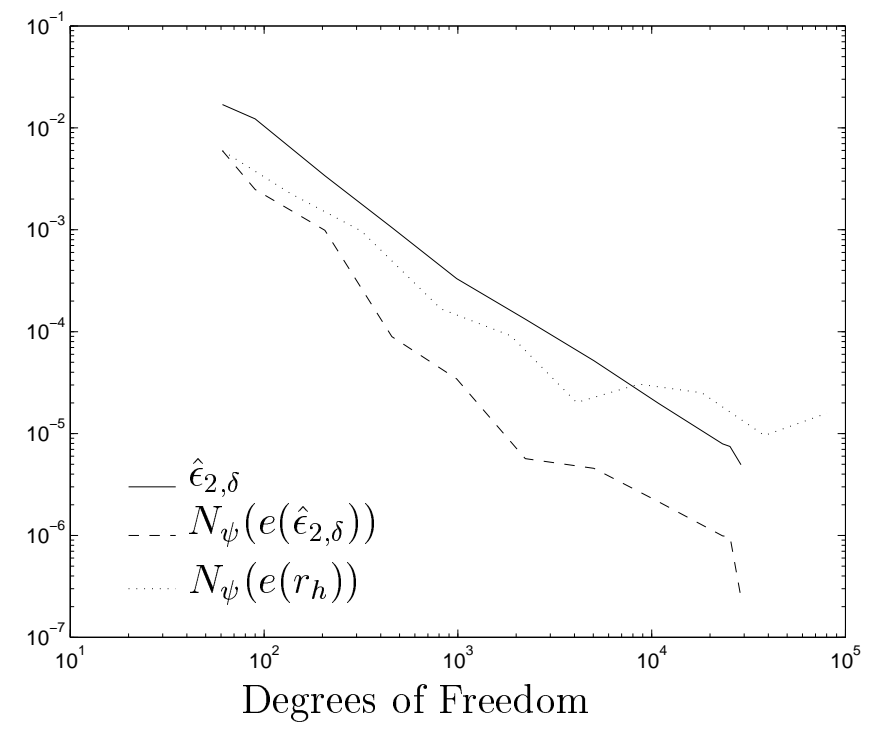

Figure 2: Example 3. Adaptive algorithm with $T O L=5.0 \times 10^{-6}$ and $\delta=h / 4$.

vicinity of the discontinuities in this problem, cf. Figure 1(b), the local weighting terms involving the approximation error between $z_{\delta}$ and $z_{\delta, h}$ are not active within these regions of the computational domain since the dual solution is extremely smooth with 'small' support concentrated near the boundary $y=1$, cf. Figure $1(\mathrm{c})$. This clearly highlights the benefit of keeping the local weighting terms $\omega_{\kappa}^{(i)}, i=1,2,3$, inside the a posteriori error bound (4.3), rather than bounding them above by a global constant.

In Figure 2 we show the performance of the adaptive algorithm described in Section 4.2 for $T O L=5.0 \times 10^{-6}$; here, the initial meshes used for the numerical solution of the primal problem and the stabilisation-dependent dual problem are shown in Figures 3(a) and $3(\mathrm{~b})$, respectively. We clearly observe in Figure 2 that even though the solution to the stabilisation-dependent dual problem has been numerically approximated, the a posteriori error estimator $\hat{\epsilon}_{2, \delta}$ remains an upper bound on the true error in the functional $N_{\psi}(\cdot)$; for clarity, we write $N_{\psi}\left(e\left(\hat{\epsilon}_{2, \delta}\right)\right)$ to denote the error in the outward normal flux on the sequence of meshes generated using the optimised mesh strategy (4.5). Additionally, in Figure 2 we plot the true error in the functional $N_{\psi}(\cdot)$ based on a sequence of meshes generated using the energy error indicator $\left\|r_{h}\right\|_{L_{2}(\kappa)}$ on each element $\kappa$ in the mesh $\mathcal{T}_{h}$ with the fixed fraction refinement strategy, cf. [13]; we denote this by $N_{\psi}\left(e\left(r_{h}\right)\right)$. Here, we see that $N_{\psi}\left(e\left(r_{h}\right)\right)$ is always inferior to $N_{\psi}\left(e\left(\hat{\epsilon}_{2, \delta}\right)\right)$; moreover, on the sequence of meshes generated by using the energy error indicator, the error in the outward normal flux starts to 'level off' as the number of degrees of freedom in the mesh increases. This indicates that by using an $a d$ hoc mesh refinement strategy only a limited amount of accuracy may be achieved in the functional of interest.

In Figures 3(c) and 3(d) we show the meshes generated for the primal and dual solution, respectively, using the adaptive algorithm outlined in Section 4.2 for $T O L=5.0 \times 10^{-5}$. Here, the mesh for the primal problem is concentrated in the upper region of the domain 


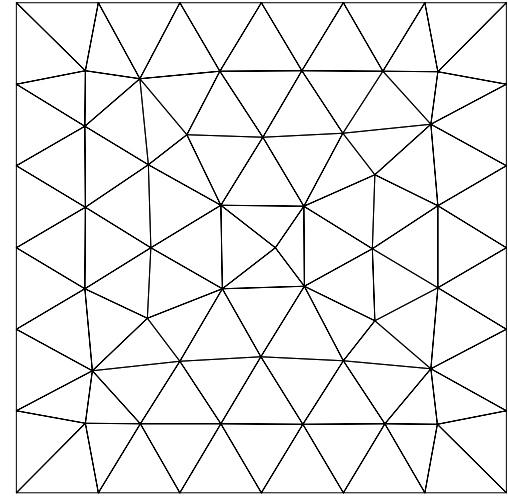

(a)

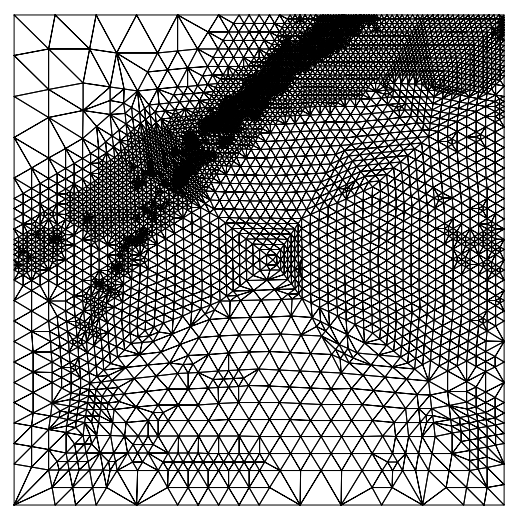

(c)

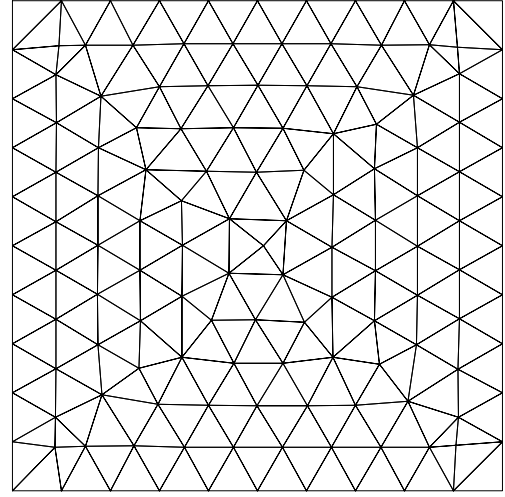

(b)

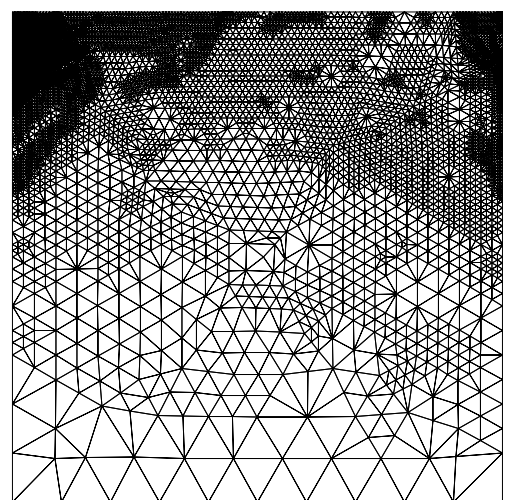

(d)

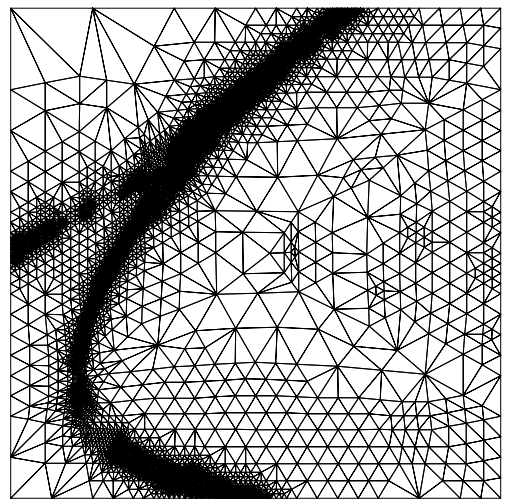

(e)

Figure 3: Example 3. (a) Initial mesh for primal problem with 61 nodes and 96 elements; (b) Initial mesh for stabilisation-dependent dual problem with 137 nodes and 232 elements; (c) Primal mesh using optimal mesh strategy (4.5) for TOL $=5.0 \times 10^{-5}$ with 5648 nodes and 11132 elements $\left(\left|N_{\psi}\left(u-u_{h}\right)\right|=6.764 \times 10^{-6}\right)$; (d) Dual mesh with 7594 nodes and 14199 elements; (e) Mesh constructed using energy error indicator with 8607 nodes and 17038 elements $\left(\left|N_{\psi}\left(u-u_{h}\right)\right|=3.057 \times 10^{-5}\right)$. 
close to the outflow boundary. Most notable is the lack of mesh refinement in the discontinuities as they enter the domain from $y=0$ and $x=0$. The mesh constructed for the stabilisation-dependent dual problem is again denser in the upper half of $\Omega$, with most of the elements concentrated near the corners $(x, y)=(0,1)$ and $(x, y)=(1,1)$ due to the presence of a boundary layer of thickness $\approx|\hat{\beta} \cdot \nu| \delta$ in the dual solution $z_{\delta}$ along $\Gamma_{-}$; here $\hat{\beta}$ denotes the normalised velocity vector. Finally, in Figure $3(\mathrm{e})$ we show the mesh constructed using the $L_{2}(\kappa)$ norm of the residual; as expected most of the elements are concentrated in the discontinuities, leading to unnecessary over-refinement.

\section{Concluding remarks}

In this article we have developed the a posteriori error analysis of stabilised finite element approximations to transport problems via duality arguments. In particular, by using a stabilisation-dependent dual problem which respects the particular structure of the Galerkin method employed, optimal error bounds for both norms and linear functionals of the approximation error were established. In the context of estimating the outward normal flux of the solution, we have implemented an adaptive algorithm capable of delivering guaranteed error control to within a user-defined tolerance TOL. Here, mesh adaptivity was employed for the numerical estimation of both the primal and dual problems; for the former problem the mesh was constructed using the optimised mesh strategy outlined in [13].

The present theory extends directly to multi-dimensional symmetric positive systems in the sense of Friedrichs (cf. [6]). Further extensions to nonlinear hyperbolic conservation laws, based on a suitable linearisation of the primal problem are possible; this work is part of our current research programme and will be presented elsewhere.

\section{References}

[1] C. Baiocchi AND F. BrezzI, Stabilization of unstable numerical methods. Current problems of analysis and mathematical physics (Italian) (Taormina, 1992), 59-63, Univ. Roma "La Sapienza", Rome, 1993.

[2] R. Becker AND R. RANNACheR, Weighted a posteriori error control in FE methods, ENUMATH-95, Paris, 18-22 Sept., 1995, in Proc. ENUMATH-97 (H.G. Bock, et al., eds.), pp. 621-637, World Scientific Publishing, Singapore, 1998.

[3] R. Becker And R. RANnAcher, A feed-back approach to error control in finite element methods: Basic analysis and examples, EAST-WEST J. Numer. Math 4, 237-264 (1996).

[4] P. G. Ciarlet, The Finite Element Method for Elliptic Problems, Studies in Mathematics and its Applications, Vol. 4. North-Holland Publishing Co., Amsterdam-New York-Oxford, 1978. xix+530 pp. ISBN: 0-444-85028-7.

[5] K. Eriksson, D. Estep, P. Hansbo, And C. Johnson, Introduction to adaptive methods for differential equations, Acta Numerica, 105-159,1995. 
[6] P. Houston, J. Mackenzie, E. Süli, and G. Warnecke, A posteriori error analysis for numerical approximations of Friedrichs systems. Numer. Math. (To appear).

[7] P. Houston And E. SÜLI, Stabilised finite element approximation of partial differential equations with nonnegative characteristic form. In preparation.

[8] P. Houston And E. SÜLI, Local mesh design for the numerical solution of hyperbolic problems. In M. Baines, editor, Numerical Methods for Fluid Dynamics VI, pp 17-30. ICFD, 1998.

[9] C. Johnson, Finite Element Methods for Partial Differential Equations, Cambridge University Press, (1993).

[10] C. Johnson, U. NÄvert and J. PitkÄranta, Finite Element Methods for linear hyperbolic problems Comp. Meth. Appl. Mech. Engrg., Vol. 45, (1984), pp. 285-312.

[11] O.OleiniK And E. V. Radkevič, Second Order Equations with Nonnegative Characteristic Form. Translated from the Russian by Paul C. Fife. Plenum Press, New York-London, 1973. vii+259 pp. ISBN: 0-306-30751-0.

[12] R. RANNACHER, A posteriori error estimation in least-squares stabilized finite element schemes, Comp. Meth. Appl. Mech. Engrg. 166, 99-114 (1998)

[13] R. Rannacher, Adaptive finite element methods, Proc. NATO-Summer School "Error Control and Adaptivity in Scientific Computing", Antalya, Aug. 9-21, 1998, Elsevier, to appear.

[14] J. RAUCH, $L_{2}$ is a continuable initial condition for Kreiss' mixed problems. Comm. Pure Appl. Math., Vol. 25, (1972), pp. 265-285.

[15] E. SüLI, A posteriori error analysis and adaptivity for finite element approximations of hyperbolic problems. In: D. Kröner, editor, Theory and Numerics of Conservations Laws, pages 123-194. Springer-Verlag, 1998. 